\title{
ASYMPTOTICS OF THE SOLUTION \\ OF THE PROBLEM OF DEFORMATION OF AN ARBITRARY LOCALLY PERIODIC THIN PLATE
}

\author{
E. A. AKIMOVA, S. A. NAZAROV, AND G. A. CHECHKIN
}

\begin{abstract}
This paper is concerned with a problem in the theory of elasticity for a thin composite plate. The principal terms are constructed for the asymptotics of the solution, with only local periodicity of the elastic moduli of the material and the shape of the plate assumed. The asymptotic expression is justified with the help of a weighted anisotropic Korn's inequality, which is proved by means of the "tetris" procedure for constructing a supporting set.
\end{abstract}

\section{CONTENTS}

1. Introduction and statement of the problem

2. Weighted anisotropic Korn's inequality

3. Procedure for constructing the formal asymptotics

4. Proof of the asymptotic expression

\section{Introduction AND STATEMENT OF THE PROBLEM}

1.1. Introduction. This paper is concerned with the construction and justification of an asymptotic expression for the solution of the problem of deformation of a thin composite plate. In contrast to previous investigations the problem is treated here in the most general setting: both the elastic properties of the material of the plate and its geometric shape are assumed to be locally periodic.

The history of the mathematical theory of plates (in contrast to the technical theories, which are numerous and often mutually contradictory, but which began more than one and a half centuries ago in the work of S. Germain and G. Kirchhoff) is not very long nor very rich in publications. The pioneering papers of Morgenstern [1] and Shoikhet [2], in which the problem of a rigorous justification of Kirchhoff's theory of plates was first posed and partially solved, appeared only about 40 years ago. This approach to the estimation of errors in the two-dimensional models of plates found its continuation in the papers [3]-[12], among others. The list contains mainly publications relating to plates whose characteristics vary smoothly in the longitudinal directions. The most complete result for such plates was established in Chapter 4 of [13: arbitrary anisotropy and inhomogeneity of the material was allowed, as well as variable thickness (laminated plates and sloping shells are included), explicit formulas were obtained for the effective moduli in the two-dimensional model, and in the weighted anisotropic norm an error bound $O\left(h^{1 / 2}\right)$ was obtained that is sharp both with respect to the exponent $1 / 2$ of the small parameter $h \in(0,1]$ (with respect to the thickness of the plate) and with respect

2000 Mathematics Subject Classification. Primary 74K20, 35B40; Secondary 35J55, 74B15, 74E30, 74E10, 35Q72.

The work of S. A. Nazarov and G. A. Chechkin was supported in part by the Russian Foundation for Basic Research (grants no. 00-01-00455 and 02-01-00693, and 02-01-00868, respectively). 
to the differentiability properties of the right-hand sides of the problem (the external loads).

References to the mathematical theory of composite plates should start with the results of Caillerie ([14], [15]) and Kohn and Vogelius ([16]-[18]) (obtained independently and published almost simultaneously) dealing with cylindrical plates manufactured from periodic composites: materials with rapidly oscillating elastic properties. Theorems on convergence of normalized elastic fields were proved in these papers under certain restrictions (we note at once that in subsequent publications ([13], [21]-[26], and others) the restrictions on the geometric level were removed only in part). It should be underscored that, first, passing to the limit leads to a loss of information about the correct asymptotic behavior of the stresses and strains in a three-dimensional plate (see the comments in [15], [18], and also [13, §6.2]) and, second, the method developed nevertheless does not enable us to derive a qualified error estimate for the two-dimensional model constructed. Such an estimate was established in [13, Ch. 6], but the proposed method of verifying it required assumptions about the strict periodicity of the plate, the introduction of essential restrictions on the smoothness of the right-hand sides, and the proof of a weighted anisotropic Korn's inequality, accompanied by subsequent conditions on the form of a periodicity cell. In this paper we remove all these conditions with the exception of the increased smoothness of the problem data.

It was apparently first understood in 2] that in the theory of thin plates it is useful to equip the Sobolev space $H^{1}\left(\Omega_{h}\right)^{3}$ with a norm that depends on the parameter $h$, is anisotropic, and distinguishes the longitudinal and transversal directions in the plate. In [27] the norms were taken to be weighted on account of the distribution (again isotropic) of the powers of the factor $h+\operatorname{dist}\left(x, \Gamma_{h}\right)$, which contains the distance to the clamped surface $\Gamma_{h}$, where Dirichlet conditions are prescribed. The approach in [27] led to a proof of anisotropic weighted Korn's inequalities for weakly bent plates [12], for plates with a periodic base [28], and under certain restrictions for perforated and corrugated plates [13] $\S 3.3]$. The paper [27] itself and its mentioned continuations exploited the possibility of cutting out a "supporting" cylindrical plate (possibly thinner but solid and penetrating the original plate), establishing the inequality on the supporting set, and then "lifting" it to the whole plate (in 12 the supporting set was composed of fragments of plates rotated with respect to each other through small angles; this method is reproduced in 2.2). It is quite clear that for an arbitrary periodicity cell a supporting plate may fail to exist, and in the case of a strongly corrugated mean surface the method of "filling" voids by using a suitable extension operator, which is a standard method in the theory of averaging and is used in [13, $\S 3.3]$ to prove Korn's inequality, may not work.

In $\S 2$ we prove a weighted anisotropic Korn's inequality on a thin locally periodic plate $\Omega_{h}$ not subject to any geometric constraints. For this purpose we use the procedure announced in 29] for constructing a supporting set. In the case of a strictly periodic plate it consists of standard elements-(Greek) crosses, beams, and tiles - and recalls the computer game "tetris". The displacement field is given on the supporting set in such a way that the corresponding elastic energy functional is bounded above by the energy functional $\mathcal{E}\left(u ; \Omega_{h}\right)$. Subsequent use of results and techniques in [12, 27] enables us to carry Korn's inequality over from the crosses to the whole plate.

In $\S 3$ we construct a formal asymptotic expression for the solution of a boundaryvalue problem in the theory of elasticity in a domain $\Omega_{h}$. A key point is Lemma 3.2, which connects the derivatives of the integral over a variable cell with slow variability of the outward normal (cf. the formulas (3.2) and (3.26)). It is this lemma that includes the main distinction from [13, $\S 6.2]$ in the derivation of the resulting problem (3.40), (3.42). We underscore that everywhere in what follows we express the defining relations of 
linearized elasticity theory using the matrix notation (and not tensor notation) proposed by S. G. Lekhnitskiu in the 1930s and given its definitive form in [13].

The fourth and last section of the paper is devoted to a justification of the asymptotic formulas constructed. The central point of our scheme of proof is a verification that the composite function $\mathcal{F}_{3}\left(y, h^{-1} x\right)$, which has zero mean with respect to the fast variable $\xi=h^{-1} x$ for fixed slow variables $y=\left(y_{1}, y_{2}\right)$, has a sufficiently small integral over a periodicity cell. In (4.29), (4.30) this is done under the condition that the function $\mathcal{F}_{3}$ belongs to the Sobolev class $H^{\gamma}$ with respect to the variable $y$ and to the class $L_{\infty}$ with respect to the variable $\xi$. The latter explains the requirements (4.1) imposed on the right-hand sides of the problem, and because of the loss of the Fredholm property by the operator of the elliptic boundary-value problem in the classes $H_{\infty}^{l}$ it is necessary to assume that the dependences on the variables $\xi$ are Hölder with arbitrarily small exponent $\alpha>0$.

The final Theorem 4.1 establishes an error estimate with majorant $O\left(h^{\gamma}\right)$, where $\gamma \in(0,1 / 2]$ is the Sobolev smoothness exponent indicated in the preceding paragraph. If we do not assume oscillation in the longitudinal directions and use the results in [11, 12] on laminated plates, then the smoothness requirements (4.1) turn out to be strongly excessive (theoretically, the Sobolev exponents can be decreased by $1+\gamma$ ). As in [13, Ch.6] for the case of strictly periodic plates, the question of the possibility of weakening the restrictions on the differentiability properties of the right-hand sides while maintaining an acceptable error estimate remains open.

1.2. Locally periodic plate. Let $S$ be a closed subset of the prism

$$
P=\left\{\xi=(\eta, \zeta) \in \mathbb{R}^{3}: 0 \leq \eta_{1} \leq l_{1}, 0 \leq \eta_{2} \leq l_{2}\right\},
$$

and let $\varphi_{y}: P \longrightarrow P$ be a family of diffeomorphisms depending smoothly on the point $y \in \bar{\omega}:=\omega \cup \partial \omega$, where $\omega$ is a domain in the plane $\mathbb{R}^{2}$ bounded by a simple closed contour $\partial \omega$. The periodic plate $\Omega_{h}$ and its lateral surface $\Gamma_{h}$ are defined by the formulas

$$
\Omega_{h}=\left\{x=(y, z): y \in \omega, \frac{x}{h} \in S(y)\right\}, \quad \Gamma_{h}=\left\{x: y \in \partial \omega, \frac{x}{h} \in S(y)\right\},
$$

where $S(y)=\varphi_{y} S$ and $h \in(0,1]$. If $P$ is the identity mapping, then the formula (1.2) determines a strictly periodic plate. Let $\Sigma(y)=\varphi_{y}(\partial S \backslash \partial P)$. We assume that the set $\Pi_{h}=\left\{x: h^{-1} x \in S(y), y \in \mathbb{R}^{2}\right\}$ is connected. The smoothness of the boundaries $\partial \Pi_{h}$ and $\partial \omega$ will be made more precise as necessary. The size $h$ is taken as a small parameter, and the thin plate $\Omega_{h}$ is clamped along its lateral surface $\Gamma_{h}$.

1.3. The problem of the theory of elasticity. We consider the field of displacements $u \in \stackrel{\circ}{H}^{1}\left(\Omega_{h} ; \Gamma_{h}\right)^{3}$. Since it vanishes on $\Gamma_{h}$, the field $u$ can be extended by zero to the layer $\Pi_{h}$. We adopt matrix notation in the defining relations of the linear theory of elasticity. The vector of displacements will be interpreted as the column $u=\left(u_{1}, u_{2}, u_{3}\right)^{\top}$ ( $\top$ is the symbol for transposition). The column of strains

$$
\varepsilon(u)=\left(\varepsilon_{11}(u), \varepsilon_{22}(u), \alpha^{-1} \varepsilon_{21}(u), \alpha^{-1} \varepsilon_{13}(u), \alpha^{-1} \varepsilon_{32}(u), \varepsilon_{33}(u)\right)^{\top}
$$

is computed by the formula $\varepsilon(u)=D\left(\nabla_{x}\right) u$, in which $D\left(\nabla_{x}\right)$ is a $6 \times 3$ matrix of first-order differential operators:

$$
D\left(\nabla_{x}\right)=\left(\begin{array}{cccccc}
\partial_{1} & 0 & \alpha \partial_{2} & \alpha \partial_{z} & 0 & 0 \\
0 & \partial_{2} & \alpha \partial_{1} & 0 & \alpha \partial_{z} & 0 \\
0 & 0 & 0 & \alpha \partial_{1} & \alpha \partial_{2} & \partial_{z}
\end{array}\right)^{\top}
$$


here $\partial_{i}=\frac{\partial}{\partial y_{i}}, \partial_{z}=\frac{\partial}{\partial z}$, and $\alpha=\frac{1}{\sqrt{2}}$. From the moduli of elasticity of the plate's material we form a positive-definite symmetric $6 \times 6$ matrix-valued function $A$ depending on the slow variables $y$ and the fast variables $\xi$; here $\xi=\left(\eta_{1}, \eta_{2}, \zeta\right)$ and $\eta_{i}=\frac{y_{i}}{h}, \zeta=\frac{z}{h}$.

The column of stresses, whose construction is analogous to that of the column of strains, has the form $\sigma(u)=A D\left(\nabla_{x}\right) u$, and the mixed boundary-value problem of the theory of elasticity can be written as

$$
\begin{aligned}
D\left(-\nabla_{x}\right)^{\top} A(\xi, y) D\left(\nabla_{x}\right) u(h, x) & =f(h, x), & & x \in \Omega_{h}, \\
D(n)^{\top} A(\xi, y) D\left(\nabla_{x}\right) u(h, x) & =g(h, x), & & x \in \partial \Omega_{h} \backslash \Gamma_{h}, \\
u(h, x) & =0, & & x \in \Gamma_{h} .
\end{aligned}
$$

Here $f$ and $g$ are the columns of volume forces and surface stresses, and $n$ is the unit (column) vector of the outward normal to the "bases" of the plate $\Sigma_{h}:=\partial \Omega_{h} \backslash \Gamma_{h}$.

If $u$ and $v$ are smooth vector-valued functions and $v$ satisfies the Dirichlet condition (1.6), then Green's formula holds:

$$
\begin{aligned}
\left(D\left(-\nabla_{x}\right)^{\top} A D\left(\nabla_{x}\right) u, v\right)_{\Omega_{h}}+\left(D(n)^{\top} A D\right. & \left.\left(\nabla_{x}\right) u, v\right)_{\Sigma_{h}} \\
& =2 \mathcal{E}\left(u, v ; \Omega_{h}\right):=\left(A D\left(\nabla_{x}\right) u, D\left(\nabla_{x}\right) v\right)_{\Omega_{h}},
\end{aligned}
$$

where $(\cdot, \cdot)_{\Theta}$ is the inner product in the space $L_{2}(\Theta)$, scalar or vector, and the functional $\mathcal{E}\left(u, v ; \Omega_{h}\right) \geq 0$ has the meaning of the elastic energy stored by the body $\Omega_{h}$. Since the matrix $A$ is positive definite,

$$
\mathcal{E}\left(u, u ; \Omega_{h}\right) \geq C_{A}\left\|D\left(\nabla_{x}\right) u ; L_{2}\left(\Omega_{h}\right)\right\|^{2} .
$$

Korn's inequality, which ensures the solvability of the problem, will be proved in the next section.

\section{Weighted Anisotropic Korn's InEQuality}

2.1. Periodic plate and auxiliary constructions. Before considering the locally periodic case, we prove Korn's inequality for a periodic plate.

Let $S(m)$ denote an integral shift of the cell $S$ (see (1.1) ) along the $\eta$-plane, that is, $S(m)=\left\{x:\left(\eta_{1}+m_{1} l_{1}, \eta_{2}+m_{2} l_{2}, \zeta\right) \in S\right\}$. Assume that the set $\widehat{\Pi}$ of interior points of the union of $S(m)$ over all $m=\left(m_{1}, m_{2}\right) \in \mathbb{Z}^{2}$ is connected and has a piecewise smooth boundary $\partial \widehat{\Pi}$. Let $\widehat{\Pi}_{h}$ be the result of contracting the set $\widehat{\Pi}$ by a factor of $h$ in all directions. As before, $\omega$ is a domain in the plane $\mathbb{R}^{2}$, and $\partial \omega$ is a piecewise smooth contour. The periodic plate $\widehat{\Omega}_{h}$ and its lateral surface $\widehat{\Gamma}_{h}$ are determined by the formulas (cf. (1.2) )

$$
\widehat{\Omega}_{h}=\left\{x \in \widehat{\Pi}_{h}: y \in \omega\right\}, \quad \widehat{\Gamma}_{h}=\left\{x \in \widehat{\Pi}_{h}: y \in \partial \omega\right\} .
$$

Remark 2.1. In 2.1 the set $\widehat{\Gamma}_{h}$ along which the plate is clamped lies on the cylindrical surface $\partial \omega \times \mathbb{R}$; however, as in [12], 13], it is possible to introduce sufficiently arbitrary small perturbations of the edge of the plate. In particular, small connected components can be removed from the set $\widehat{\Omega}_{h}$ in (2.1) (pieces of growths "accidentally" cut off by the surface $\partial \omega \times \mathbb{R})$.

According to (2.1) the field $u \in \stackrel{\circ}{H}^{1}\left(\widehat{\Omega}_{h} ; \widehat{\Gamma}_{h}\right)^{3}$ can be extended by zero to the periodic layer $\widehat{\Pi}_{h}$.

We carry out the constructions in the layer $\widehat{\Pi}$ of unit "thickness" and at the very end perform an $h$-contraction. There is a plane $\left\{\xi: \zeta=\zeta_{0}\right\}$ whose intersection with $\widehat{\Pi}$ contains the points $P^{m}=\left(a_{1}+m_{1} l_{1}, a_{2}+m_{2} l_{2}, \zeta_{0}\right)$ for all $m \in \mathbb{Z}^{2}$ and some $a \in \mathbb{R}^{2}$. 


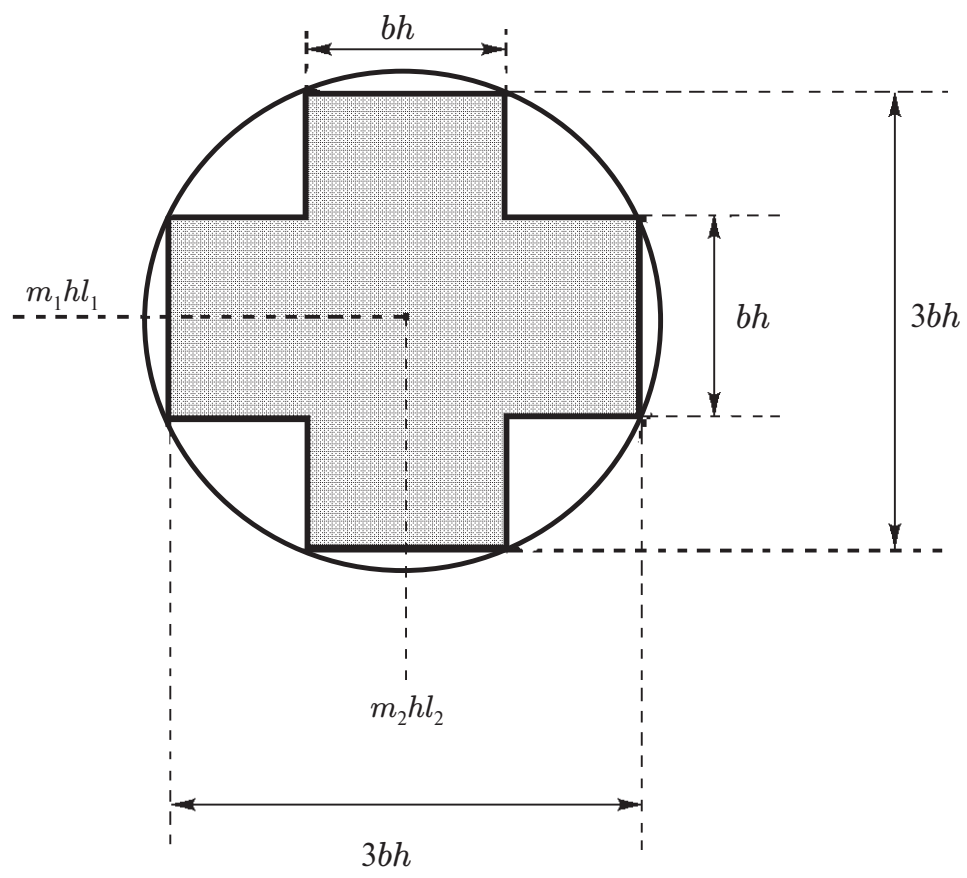

FiguRE 1. The ball $\mathbf{B}_{r}^{m}$ containing the cross $\mathbf{K}^{m}$.

Furthermore, there is an $r>0$ such that the ball $\mathbf{B}_{r}^{m}$ with center $P^{m}$ and radius $r$ is interior to $\widehat{\Pi}$. In each ball $\mathbf{B}_{r}^{m}$ we can inscribe a cross $\mathbf{K}^{m}$-a union of two parallelepipeds

$$
\left\{\xi:\left|\eta_{i}-P_{i}^{m}\right|<3 b,\left|\eta_{3-i}-P_{3-i}^{m}\right|<b,\left|\zeta-\zeta_{0}\right|<b\right\}, \quad i=1,2
$$

(see Figure 1); the exact connection of the quantity $b>0$ with the radius $r$ is not needed.

We consider four neighboring crosses $\mathbf{K}^{(1)}, \mathbf{K}^{(2)}, \mathbf{K}^{(3)}$, and $\mathbf{K}^{(4)}=\mathbf{K}^{(0)}$ (their centers are at the vertices of an $l_{1} \times l_{2}$ rectangle). The four pairs of parallelepipeds (2.2) forming these crosses have square faces "looking" at each other. We extend the larger faces of the parallelepipeds and form four beams $\mathbf{Q}^{(1)}, \ldots, \mathbf{Q}^{(4)}$ joining the pairs of crosses (see Figure 2). Finally, the crosses and beams bound a tile $\mathbf{F}$ in the layer $\mathbf{L}=\left\{\xi:\left|\zeta-\zeta_{0}\right|<b\right\}$. Thus, we have succeeded in paving the layer $\mathbf{L}$ with standard bodies: crosses, beams, and tiles.

Each cross can be formed from five cubes: a central cube and four lateral cubes. If the beam $\mathbf{Q}^{(j)}$ joining lateral cubes of neighboring crosses $\mathbf{K}^{(j)}$ and $\mathbf{K}^{(j+1)}$ goes outside the periodic layer $\widehat{\Pi}$, then we can form a connected set $\mathcal{Q}^{(j)}$ lying in $\Pi$, intersecting the crosses in the indicated cubes, and called a yoke (Figure 2). It suffices to fix a pair of yokes and replicate them by periodicity. We stress that $\mathcal{Q}^{(j)}=\mathbf{Q}^{(j)}$ in the case $\mathbf{Q}^{(j)} \subset \widehat{\Pi}$.

We now carry out an $h$-contraction and add the index $h$ to the notation for the geometric objects constructed in this subsection.

2.2. Korn's inequality on a periodic plate. We introduce a functional similar to the elastic energy,

$$
\mathcal{E}\left(u ; \widehat{\Omega}_{h}\right):=\mathcal{E}\left(u, u ; \widehat{\Omega}_{h}\right)=\sum_{p, q=1}^{3} \int_{\widehat{\Omega}_{h}}\left|\frac{\partial u_{p}}{\partial x_{q}}(x)+\frac{\partial u_{q}}{\partial x_{p}}(x)\right|^{2} d x
$$




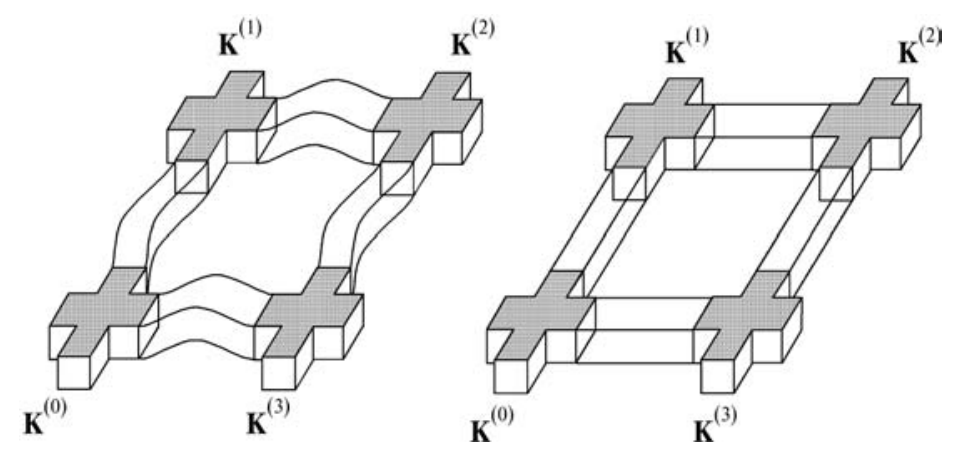

FIgURE 2. A construction containing yokes (left) and beams (right).

and a weighted anisotropic norm $|\bullet|$ in the space $\stackrel{\circ}{H^{1}}\left(\widehat{\Omega}_{h} ; \Gamma_{h}\right)^{3}$ distinguishing between the longitudinal directions $y_{i}=x_{i}$ and the transversal direction $z=x_{3}$ in the plate,

$$
\begin{aligned}
\left|u ; \widehat{\Omega}_{h}\right|^{2}=\int_{\widehat{\Omega}_{h}}\left\{\sum _ { i = 1 } ^ { 2 } \left[\left|\nabla_{y} u_{i}\right|^{2}+h^{2} \rho_{h}^{-2}\left(\left|\frac{\partial u_{i}}{\partial z}\right|^{2}\right.\right.\right. & \left.\left.+\left|\frac{\partial u_{3}}{\partial y_{i}}\right|^{2}\right)+\rho_{h}^{-2}\left|u_{i}\right|^{2}\right] \\
& \left.+\left|\partial_{z} u_{3}\right|^{2}+h^{2} \rho_{h}^{-4}\left|u_{3}\right|^{2}\right\} d x .
\end{aligned}
$$

The weight factor $\rho_{h}(x)=h+\operatorname{dist}\left\{x, \widehat{\Gamma}_{h}\right\}$ acquires the order of the thickness $h$ near the plate's edge $\widehat{\Gamma}_{h}$, on which the field $u$ vanishes.

Theorem 2.1. If $u \in \stackrel{\circ}{H^{1}}\left(\widehat{\Omega}_{h} ; \widehat{\Gamma}_{h}\right)^{3}$, then the weighted Korn's inequality

$$
\left|u ; \widehat{\Omega}_{h}\right|^{2} \leq C \mathcal{E}\left(u ; \widehat{\Omega}_{h}\right)
$$

is valid, with a constant $C$ independent of the field $u$ and the parameter $h \in(0,1]$.

We break up the proof of Theorem 2.1 into several steps. In the first step we construct a completion $\widetilde{u} \in H^{1}\left(\mathbf{L}_{h}\right)^{3}$ of the restriction of the field $u$ to the crosses while maintaining the estimate

$$
\mathcal{E}\left(\widetilde{u} ; \mathbf{L}_{h}\right) \leq c \mathcal{E}\left(u ; \widehat{\Pi}_{h}\right)
$$

we recall that $u=0$ outside $\widehat{\Pi}_{h} \backslash\left(\omega \times \mathbb{R}^{2}\right)$. Since according to [27] (see also [13]) $\left|\widetilde{u} ; \mathbf{L}_{h}\right|^{2} \leq c \mathcal{E}\left(\widetilde{u} ; \mathbf{L}_{h}\right)$ for the weighted norm (2.3), Korn's inequality (2.4) is obtained by summing the inequalities

$$
\mathbf{|} \tilde{u} ;\left.S_{h}^{(m)}\right|^{2} \leq c\left(\mathcal{E}\left(\widetilde{u} ; S_{h}^{(m)}\right)+\left|u ; \mathbf{K}_{h}^{(m)}\right|^{2}\right)
$$

(see [12], 13]) for the cells $S_{h}^{(m)}$ that intersect $\widehat{\Omega}_{h}$ and then using the estimate (2.5).

We carry out the required completion $\widetilde{u}$. Let us consider the beam $\mathbf{Q}_{h}^{(j)}$ joining the crosses $\mathbf{K}^{(j)}$ and $\mathbf{K}^{(j+1)}$. The proof of the next assertion is essentially contained in [30] (the only novelty is a dilation of the coordinates).

Lemma 2.1. There exists an operator $T$ of extension from the yoke $\mathcal{Q}_{h}^{(j)}$ to the set $\mathcal{Q}_{h}^{(j)} \cup \mathbf{Q}_{h}^{(j)}$ in the class $H^{1}$, and

$$
\mathcal{E}\left(T u ; \mathcal{Q}_{h}^{(j)} \cup \mathbf{Q}_{h}^{(j)}\right) \leq c \mathcal{E}\left(u ; \mathcal{Q}_{h}^{(j)}\right)
$$


with a constant $c$ independent of the field $u \in H^{1}\left(\mathcal{Q}_{h}^{(j)}\right)^{3}$ and the geometric parameter $h \in(0,1]$.

Since there are two types of yokes, we use two extension operators and with their help find the field $\widetilde{u}$ on the beams $\mathbf{Q}_{h}^{(m)}$. By construction, the beams do not come into contact, and hence $\widetilde{u}$, which coincides with $u$ on the crosses, falls in the class $H^{1}$ on the frame construction (the layer $\mathbf{L}$ with the tiles cut out). It remains to use the analogue of Lemma 2.1 and extend the field $\widetilde{u}$ from the frame to the tiles (which do not meet!).

We underscore that the field $\widetilde{u}$ is not in general an extension of the field $u$, because it can differ from the latter on the tiles.

We sketch the subsequent scheme of the proof. Let $\left\{\omega^{1}, \ldots, \omega^{N}\right\}$ be a fixed (independent of $h$ ) covering of the domain $\omega$. We first choose domains $\omega^{1}, \ldots, \omega^{K}$ intersecting the contour $\partial \omega$. For "straight" plates

$$
G_{h}^{i}=\left\{x: y \in \omega^{i},-\frac{b h}{2}<z<\frac{b h}{2}\right\}
$$

the weighted Korn's inequality of the form (2.4) in [27] is applicable. Its validity is ensured by the Dirichlet conditions on $\widehat{\Gamma}_{h}^{0}$. These inequalities are then extended to the "curved" plates $\widehat{\Omega}_{h}^{i}=\left\{x \in \widehat{\Omega}_{h}: y \in \omega^{i}\right\}, i=1, \ldots, K$. This yields the weighted inequality (2.4), restricted to a fixed neighborhood of the lateral surface of the plate $\widehat{\Omega}_{h}$. Next we consider the domains $\omega^{K+1}, \ldots, \omega^{L}$ which intersect at least one of the sets $\omega^{1}, \ldots, \omega^{K}$. Since $\rho_{h}(y)>C>0$ for $y \in \omega^{K+1} \cup \cdots \cup \omega^{L}$, the inequalities (2.4) on $G_{h}^{K+1}, \ldots, G_{h}^{L}$ cease to be weighted in essence, and can be established with the help of a result in [2]. It will be proved that $G_{h}^{n}$ can be replaced by $\widehat{\Omega}_{h}^{n}, n=K+1, \ldots, L$, and still more can be moved away from the edge of the plate $\widehat{\Omega}_{h}$. The next step repeats the preceding one, and we need finitely many steps for going through all the elements of the covering $\left\{\omega^{n}\right\}_{n=1, \ldots, N}$. let

We first obtain auxiliary inequalities on the cylindrical plates $G_{h}^{n}$. For $\widetilde{u} \in H^{1}\left(G_{h}^{n}\right)^{3}$

$$
\begin{aligned}
\mathbf{|} \tilde{u} ;\left.G_{h}^{n}\right|_{1} ^{2}=\int_{G_{h}^{n}}\left\{\sum _ { i = 1 } ^ { 2 } \left[\left|\nabla_{y} \widetilde{u}_{i}\right|^{2}+h^{2}\left(\left|\frac{\partial \widetilde{u}_{i}}{\partial z}\right|^{2}+\left|\frac{\partial \widetilde{u}_{3}}{\partial y_{i}}\right|^{2}\right)\right.\right. & \left.+\left|\widetilde{u}_{i}\right|^{2}\right] \\
& \left.+\left|\frac{\partial \widetilde{u}_{3}}{\partial z}\right|^{2}+h^{2}\left|\widetilde{u}_{3}\right|^{2}\right\} d x .
\end{aligned}
$$

If in addition $\widetilde{u}=0$ on $T_{h}^{n}=\left\{x: y \in t^{n},|z|<\frac{b h}{2}\right\}$, where $t^{n}$ is a nonempty open arc on the contour $\partial \omega^{n}$, then $\left|\widetilde{u} ; G_{h}^{n}\right|_{\rho}^{2}$ is understood to be the integral (2.7), in which the powers of the weight factor $\rho_{h}(y)$ are distributed in precisely the same way as in (2.3).

Lemma 2.2. 1) For $\widetilde{u} \in \stackrel{\circ}{H^{1}}\left(G_{h}^{n}, T_{h}^{n}\right)^{3}$,

$$
\mathbf{u} ;\left.G_{h}^{n}\right|_{\rho} ^{2} \leq c \mathcal{E}\left(\widetilde{u} ; G_{h}^{n}\right) .
$$

2) If $\widetilde{u} \in H^{1}\left(G_{h}^{n}\right)^{3}$, and $\theta^{n}$ is a proper subdomain of $\omega^{n}$ and

$$
Q_{h}^{n}=\left\{x: y \in \theta^{n},|z|<\frac{b h}{2}\right\},
$$

then

$$
\mathbf{|} ;\left.G_{h}^{n}\right|_{1} ^{2} \leq c\left(E\left(\widetilde{u} ; G_{h}^{n}\right)+\left|\widetilde{u} ; Q_{h}^{n}\right|_{1}^{2}\right) .
$$

The constant $c$ in (2.8) and (2.9) is independent of $\widetilde{u}$ and $h \in(0,1)$. 
Proof. The first assertion is contained in [27]. We verify the inequality (2.9), using methods from [2]. After the changes

$$
z \longmapsto \zeta=h^{-1} z, \quad \widetilde{u}_{3} \longmapsto \widetilde{v}_{3}=h \widetilde{u}_{3}
$$

we get that for $h \in(0,1]$

$$
\begin{aligned}
& \int_{G_{h}^{n}}\left\{\sum_{i=1}^{2}\left(\left|\frac{\partial \widetilde{u}_{i}}{\partial y_{i}}\right|^{2}+\left|\frac{\partial \widetilde{u}_{i}}{\partial z}+\frac{\partial \widetilde{u}_{3}}{\partial y_{i}}\right|^{2}\right)+\left|\frac{\partial \widetilde{u}_{1}}{\partial y_{2}}+\frac{\partial \widetilde{u}_{2}}{\partial y_{1}}\right|^{2}+\left|\frac{\partial \widetilde{u}_{3}}{\partial z}\right|^{2}\right\} d y d z \\
& +h \int_{G_{h}^{n}}\left\{\sum_{i=1}^{2}\left(\left|\frac{\partial \widetilde{u}_{i}}{\partial y_{i}}\right|^{2}+h^{-2}\left|\frac{\partial \widetilde{u}_{i}}{\partial \zeta}+\frac{\partial \widetilde{v}_{3}}{\partial y_{i}}\right|^{2}\right)+\left|\frac{\partial \widetilde{u}_{1}}{\partial y_{2}}+\frac{\partial \widetilde{u}_{2}}{\partial y_{1}}\right|^{2}+h^{-4}\left|\frac{\partial \widetilde{v}_{3}}{\partial \zeta}\right|^{2}\right\} d y d \zeta \\
& \geq h \int_{G_{h}^{n}}\left\{\sum_{i=1}^{2}\left(\left|\frac{\partial \widetilde{u}_{i}}{\partial y_{i}}\right|^{2}+\left|\frac{\partial \widetilde{u}_{i}}{\partial \zeta}+\frac{\partial \widetilde{v}_{3}}{\partial y_{i}}\right|^{2}\right)+\left|\frac{\partial \widetilde{u}_{1}}{\partial y_{2}}+\frac{\partial \widetilde{u}_{2}}{\partial y_{1}}\right|^{2}+\left|\frac{\partial \widetilde{v}_{3}}{\partial \zeta}\right|^{2}\right\} d y d \zeta .
\end{aligned}
$$

By (1.7) and (1.3) the left-hand side of (2.11) is bounded above by the quantity $c \mathcal{E}\left(\widetilde{u} ; G_{h}^{n}\right)$. The integral $I$ on the right-hand side differs from the elastic energy of the field $\left(\widetilde{u}_{1}, \widetilde{u}_{2}, \widetilde{v}_{3}\right)^{\top}$ in the body $G_{1}^{n}$ with unit dimensions only by factors that are inessential in this context. Korn's inequality on $G_{1}^{n}$ (see [31], 32], and other references) takes the form

$$
\begin{aligned}
\sum_{i=1}^{2}\left\|\widetilde{u}_{i} ; H^{1}\left(G_{1}^{n}\right)\right\|^{2}+ & \left\|\widetilde{v}_{3}^{n} ; H^{1}\left(G_{1}^{n}\right)\right\|^{2} \\
& \leq c\left(I+\sum_{i=1}^{2}\left\|\widetilde{u} ; L_{2}\left(Q_{1}^{n}\right)\right\|^{2}+\left\|\widetilde{v}_{3} ; L_{2}\left(Q_{1}^{n}\right)\right\|^{2}\right) .
\end{aligned}
$$

Of course, the constant $c$ in (2.12) is independent of $\widetilde{u}$ and $h$. We must now multiply (2.12) by $h$ and make the substitutions inverse to (2.10). The right-hand side of the inequality obtained from (2.11) and (2.12) does not exceed the right-hand side of (2.9). However, the left-hand side differs from the integral in (2.7) only in that the factor $h^{4}$ appears with the term $\left|\partial_{z} \widetilde{u}_{3}\right|^{2}$. This factor can be removed without consequences, because the term $\left|\partial_{z} \widetilde{u}_{3}\right|^{2}$ itself can be found in the first integrand in (2.11).

We consider the rectangles

$$
\left\{y:\left|y_{i}-m_{i} h l_{i}\right|<\frac{l_{i} h}{2}, m_{i} \in\{0, \pm 1, \ldots\}, i=1,2\right\} .
$$

Let $q$ be one such rectangle that intersects $\omega$; the center of the rectangle is located at a point $y^{q}$. We consider the domain

$$
Q=\widehat{\Omega}_{h} \cap\{x: y \in q, z \in \mathbb{R}\} .
$$

The restriction of the field $u \in \stackrel{\circ}{H}^{1}\left(\Omega_{h}, \Gamma_{h}\right)^{3}$ to $Q$ is representable in the form

$$
u(x)=u^{\perp}(x)+u^{0}(x),
$$

where $u^{0}(x)=d(x) a$, with $a$ a column in $\mathbb{R}^{6}$ and

$$
d(x)=\left(\begin{array}{cccccc}
0 & 1 & 0 & -\alpha z & 0 & \alpha y_{1} \\
0 & 0 & 1 & \alpha y_{2} & -\alpha y_{1} & 0
\end{array}\right) .
$$

Since $u^{0}$ is a displacement of $Q$ as a rigid entity, we have the equalities

$$
\varepsilon(u)=\varepsilon\left(u^{\perp}\right), \quad \mathcal{E}(u ; Q)=\mathcal{E}\left(u^{\perp} ; Q\right),
$$


which follow from the relations $D\left(\nabla_{x}\right)^{\top} d(x)=\mathbb{O}_{6}$ (the $6 \times 6$ zero matrix). Suppose that the ball $B$ of radius $h r$ and center on the line $y=y_{q}$ lies entirely in $Q$ and

$$
a=d_{B}^{-1} \int_{B} d(x)^{\top} u(x) d x, \quad d_{B}=\int_{B} d(x)^{\top} d(x) d x .
$$

We underscore that $d_{B}$ is a $6 \times 6$ Gram matrix and is positive definite and symmetric. Moving the origin of coordinates to the center of $B$, we find that

$$
d_{B}=\frac{4}{3} \pi r^{3} h^{3}\left(\begin{array}{cc}
\mathbb{I}_{3} & \mathbb{O}_{3} \\
\mathbb{O}_{3} & P \mathbb{I}_{3}
\end{array}\right), \quad P=\frac{1}{5} r^{2} h^{2} .
$$

Note that, by (2.13) and (2.16),

$$
\int_{Q} d(x)^{\top} u^{\perp}(x) d x=\int_{Q} d(x)^{\top} u(x) d x-\int_{Q} d(x)^{\top} d(x) d x a=d_{B} a-d_{B} a=0 .
$$

The orthogonality conditions (2.18) give us Korn's inequality

$$
\mathcal{E}\left(u^{\perp} ; Q\right) \geq c\left(\left\|\nabla_{x} u^{\perp} ; L_{2}(Q)\right\|^{2}+h^{-2}\left\|u^{\perp} ; L_{2}(Q)\right\|^{2}\right)
$$

(see, for example, 32]). We point out two facts. First, the factor $h^{-2}$ appeared in connection with the substitution $x \longmapsto \xi=h^{-1} x$, which eliminates the small parameter $h$ and translates $Q$ into the "unit" prism with curvilinear bases

$$
Q_{1}=\left\{\xi=\left(\xi^{\prime}, \xi_{3}\right) \in \widehat{\Omega}_{h}: y_{i}^{q}-\frac{h l_{i}}{2}<\xi_{i}^{\prime}<y_{i}^{q}+\frac{h l_{i}}{2}, i=1,2\right\} .
$$

Second, $Q$ can be partitioned into a finite number of domains that are starlike with respect to a ball of radius $r h$, and hence by [34, [30] the constant $c>0$ in (2.18) can be expressed in terms of the ratio of diameters $(2 h r)^{-1} \operatorname{diam} Q=(2 r)^{-1} \operatorname{diam} Q_{1}$, which does not depend on $h$ and can be taken to be common for all squares $q$ in the partition.

Lemma 2.3. For $u \in \stackrel{\circ}{H^{1}}\left(\widehat{\Omega}_{h}, \widehat{\Gamma}_{h}\right)^{3}$,

$$
\left|u ; \widetilde{\Omega}_{h}^{n}\right|^{2} \leq c\left(\left|\widetilde{u} ; G_{h}^{n}\right|^{2}+\mathcal{E}\left(u ; \widetilde{\Omega}_{h}^{n}\right)\right),
$$

where $\widetilde{\Omega}_{h}^{n}=\left\{x \in \widehat{\Omega}_{h}^{n}: y \in \omega^{n}\right.$, $\left.\operatorname{dist}\left\{y, d \omega^{n}\right\}>\sqrt{2} h\right\}$, and $\widetilde{u}$ is the completion of the function $u$ on $\mathbf{L}_{h}$ constructed above. The constant $c$ in (2.20) is independent of $u$ and $h \in(0,1]$.

Proof. Let $q$ be one of the $h l_{1} \times h l_{2}$ rectangles contained in $\omega_{n}$, let $Q$ be the corresponding prism with curvilinear base, and let $B$ be a ball such that $B \subset Q \cap G_{h}^{n}$. Since $\rho_{h}(y)^{-2} \leq$ $h^{-2},(2.15)$ and (2.19) give us the inequality

$$
\left|u^{\perp} ; Q\right|^{2} \leq c \mathcal{E}\left(u^{\perp} ; Q\right)=c \mathcal{E}(u ; Q) .
$$

Below we shall verify the following formula for the component $u^{0}(x)=d(x) a$ in the decomposition (2.13):

$$
\left|u^{0} ; Q\right|^{2} \leq c|u ; B|^{2} .
$$

The constants in (2.21) and (2.22) can be taken to be common for all squares falling in $\omega^{n}$. Therefore, by summing these inequalities we arrive at the desired relation (2.20):

$$
\begin{aligned}
\left|u ; \widetilde{\Omega}_{h}^{n}\right|^{2} & =\sum_{\ldots}|u ; Q|^{2} \leq 2\left(\sum_{\ldots}\left|u^{0} ; Q\right|^{2}+\sum_{\ldots}\left|u^{\perp} ; Q\right|^{2}\right) \\
& \leq 2 c\left(\sum_{\ldots}|u ; B|^{2}+\sum_{\ldots} \mathcal{E}(u ; Q)\right) \leq 2 c\left(\left|u ; G_{h}^{n}\right|^{2}+\mathcal{E}\left(u ; \widetilde{\Omega}_{h}^{n}\right)\right) .
\end{aligned}
$$


Thus, it remains to verify the estimate (2.22). We do this after locating the center $\left(y^{B}, z^{B}\right)$ of $B$ at the origin of coordinates (so that we do not have to write $d\left(y-y^{B}, z-z^{B}\right.$ ) everywhere instead of $d(x))$. According to (2.16),

$$
a:=\left(a_{1}, \ldots, a_{6}\right)^{\top}=d_{B}^{-1} J, \quad J:=\left(J_{1}, \ldots, J_{6}\right)^{\top}=\int_{B} d(x)^{\top} u(x) d x .
$$

Therefore, a direct computation of the norm $|d a ; B|$ using the explicit expression (2.17) for the matrix $d_{B}$ shows that

$$
\begin{aligned}
& |d a ; B|^{2} \leq c h^{3}\left\{\rho^{-2}\left(\left|a_{1}\right|^{2}+\left|a_{2}\right|^{2}\right)+h^{2} \rho^{-4}\left|a_{3}\right|^{2}+h^{2} \rho^{-2}\left(\left|a_{4}\right|^{2}+\left|a_{5}\right|^{2}\right)+\left|a_{6}\right|^{2}\right\} \\
& \leq c h^{-3}\left\{\rho^{-2}\left(\left|J_{1}\right|^{2}+\left|J_{2}\right|^{2}\right)+h^{2} \rho^{-4}\left|J_{3}\right|^{2}+h^{-2} \rho^{-2}\left(\left|J_{4}\right|^{2}+\left|J_{5}\right|^{2}\right)+h^{-4}\left|J_{6}\right|^{2}\right\}
\end{aligned}
$$

where $\rho=\rho_{h}\left(y^{q}\right)$ and there are constants $C, c>0$ such that

$$
c \rho \leq \rho_{h}(y) \leq C \rho .
$$

For $i=1,2$ we have from (2.14) and (2.24) that

$$
\left|J_{i}\right|^{2}=\left|\int_{B} u_{i} d x\right|^{2} \leq c \operatorname{meas}_{3} B \int_{B}\left|u_{i}\right|^{2} d x \leq c h^{3} \rho^{2} \int_{B} \rho_{h}^{-2}\left|u_{i}\right|^{2} d x \leq c h^{3} \rho^{2}|u ; B|^{2} .
$$

Similarly,

$$
\left|J_{3}\right|^{2} \leq \operatorname{ch}^{3} \int_{B}\left|u_{3}\right|^{2} d x \leq \operatorname{ch} \rho^{4}|u ; B|^{2} .
$$

Let $P(x)=\left|y-y^{B}\right|^{2}+\left|z-z^{B}\right|^{2}-r^{2} h^{2}$ (recall that $r h$ is the radius of the ball $B$ ). Then for $i=1,2$

$$
\begin{aligned}
\left|J_{6-i}\right|^{2} & =\frac{1}{2}\left|\int_{B}\left(z u_{i}-y_{i} u_{3}\right) d x\right|^{2}=\frac{1}{8}\left|\int_{B}\left(u_{i} \frac{\partial P}{\partial z}-u_{3} \frac{\partial P}{\partial y_{i}}\right) d x\right|^{2} \\
& =\frac{1}{8}\left|\int_{B} P\left(\frac{\partial u_{i}}{\partial z}-\frac{\partial u_{3}}{\partial y_{i}}\right) d x\right|^{2} \leq c h^{3}\left(\max _{B} P\right)^{2} \int_{B}\left(\left|\frac{\partial u_{i}}{\partial z}\right|^{2}+\left|\frac{\partial u_{3}}{\partial y_{i}}\right|^{2}\right) d x \\
& \leq \operatorname{ch}^{5} \rho^{2} \int_{B} h^{2} \rho_{h}^{-2}\left(\left|\frac{\partial u_{i}}{\partial z}\right|^{2}+\left|\frac{\partial u_{3}}{\partial y_{i}}\right|^{2}\right) d x \leq \operatorname{ch}^{5} \rho^{2}|u ; B|^{2}
\end{aligned}
$$

Finally,

$$
\begin{aligned}
\left|J_{6}\right|^{2} & =\frac{1}{2}\left|\int_{B}\left(x_{1} u_{2}-x_{2} u_{1}\right) d x\right|^{2}=\frac{1}{8}\left|\int_{B} P\left(\frac{\partial u_{1}}{\partial x_{2}}-\frac{\partial u_{2}}{\partial x_{1}}\right) d x\right|^{2} \\
& \leq \operatorname{ch}^{7} \int_{B}\left(\left|\frac{\partial u_{1}}{\partial x_{2}}\right|^{2}+\left|\frac{\partial u_{2}}{\partial x_{1}}\right|^{2}\right) d x \leq \operatorname{ch}^{7}|u ; B|^{2} .
\end{aligned}
$$

Substituting the estimates obtained for $\left|J_{1}\right|, \ldots,\left|J_{6}\right|$ in (2.23), we arrive at (2.20), which is what was required.

In conclusion we discuss the order of application of the lemmas proved. If the domain $\omega^{1}$ intersects $\partial \omega$, then in view of Lemma 2.2(1) we get for the completion of $u$ the relation

$$
\mathbf{|} \widetilde{u} ;\left.G_{h}^{1}\right|_{\rho} ^{2} \leq c \mathcal{E}\left(\widetilde{u} ; G_{h}^{1}\right) .
$$

The inequality (2.20) in Lemma 2.3 and the inequality (2.25) give us that

$$
\left|u ; \widetilde{\Omega}_{h}^{1}\right|^{2} \leq c\left(\mathcal{E}\left(\widetilde{u} ; G_{h}^{1}\right)+\mathcal{E}\left(u ; \widetilde{\Omega}_{h}^{1}\right)\right) .
$$


Suppose that the intersection $G_{h}^{1} \cap \widetilde{\Omega}_{h}^{1}$ contains a ball $B^{1}$ with radius $r_{1} h$. The classical Korn's inequality provides the two estimates

$$
\begin{aligned}
& \left\|u^{\perp} ; H^{1}\left(B^{1}\right)\right\|^{2} \leq c \mathcal{E}\left(u^{\perp} ; B^{1}\right)=c \mathcal{E}\left(u ; B^{1}\right), \\
& \left\|u^{\perp} ; H^{1}\left(\widetilde{\Omega}_{h}^{1}\right)\right\|^{2} \leq c\left(\left\|u^{\perp} ; H^{1}\left(B^{1}\right)\right\|^{2}+\mathcal{E}\left(u ; \widetilde{\Omega}_{h}^{1}\right)\right) \leq c \mathcal{E}\left(u ; \widetilde{\Omega}_{h}^{1}\right) .
\end{aligned}
$$

By the formulas

$$
\begin{aligned}
\mathcal{E}\left(\widetilde{u} ; G_{h}^{1}\right)=\mathcal{E}\left(\widetilde{u}^{\perp} ; G_{h}^{1}\right) & \leq c\left\|\widetilde{u}^{\perp} ; H^{1}\left(G_{h}^{1}\right)\right\|^{2}, \\
\left\|\widetilde{u}^{\perp} ; H^{1}\left(G_{h}^{1}\right)\right\|^{2} & \leq c\left\|u^{\perp} ; H^{1}\left(\widetilde{\Omega}_{h}^{1}\right)\right\|^{2},
\end{aligned}
$$

we now arrive at Korn's inequality on $\widetilde{\Omega}_{h}^{1}$ :

$$
\left|u ; \widetilde{\Omega}_{h}^{1}\right|^{2} \leq \mathcal{E}\left(u ; \widetilde{\Omega}_{h}^{1}\right) .
$$

Suppose that the area of the intersection of $\omega^{K+1}$ and $\omega^{1}$ is nonzero. Then Korn's inequality on $\omega^{1} \cup \omega^{K+1}$ is a consequence of Lemmas 2.2 2 ) and 2.3. All the situations that arise when we go through the elements of the covering $\left\{\omega^{1}, \ldots, \omega^{N}\right\}$ are essentially exhausted by the two mentioned.

2.3. Korn's inequality for a locally periodic plate. The procedure described in 2.2 establishes the inequality (2.4) for a strictly periodic plate. Together with this, the relation (2.6) is preserved also when the cell $S_{h}^{(m)}$ is smoothly changed (see [34] and [12], [13]). This enables to carry out a proof for locally periodic plates. We remark that the situation of the supporting layer $\mathbf{L}_{h}$ can change jump-like in the domain $\omega$, that is, in this subsection we must use in addition the method worked out in [12 for carrying the weighted Korn's inequality from one fragment of the plate to another.

Let $\Omega_{h}$ be the locally periodic plate defined in (1.2). It can be projected onto the set $\omega$ in the plane of the variables $y$. The covering $\left\{\omega^{1}, \ldots, \omega^{N}\right\}$ of $\omega$ can be chosen to be fine enough that the following conditions hold:

$1^{\circ}$ each of the sets $\Omega_{h}^{i}=\left\{x \in \Omega_{h}: y \in \omega^{i}\right\}$ contains a system $\left\{\mathbf{K}_{h, i}^{m}\right\}_{m \in A_{i}}$ of crosses that is periodic with respect to $y$, where $A_{i}$ is a subset of $\mathbb{Z}^{2}$;

$2^{\circ}$ as in 2.1, each of the crosses is a union of two parallelepipeds, but the edges of these parallelepipeds may not be parallel to the coordinate axes, in contrast to (2.2);

$3^{\circ}$ in the framework of the single system with index $i$ the crosses $\mathbf{K}_{h, i}^{m}, m \in A_{i}$, lie in the common layer $\mathbf{L}_{h, i}$ bounded by two parallel planes at a distance from each other equal to the thickness of the crosses.

From the indicated covering we now choose the domains $\omega^{1}, \ldots, \omega^{k}$ intersecting the contour $\partial \omega$, and we apply the weighted Korn's inequality to the plates $\Omega_{h}^{1}, \ldots, \Omega_{h}^{k}$. Although the plate $\Omega_{h}^{i}$ is not strictly periodic, the procedure described in 2.2 can be applied to it in view of the condition $1^{\circ}$. We join the crosses contained in $\Omega_{h}^{i}$ by beams, and we introduce the tiles bounded by these beams and the crosses (in this way the layer $\mathbf{L}_{h, i}$ in the condition $3^{\circ}$ is paved by standard bodies). Next we form yokes: connected sets lying in $\Omega_{h}^{i}$ and intersecting the crosses in lateral cubes. In contrast to the case of a periodic plate, all the yokes can turn out to be different. In this connection we need a set of operators of extension from the yokes to the beams. The rest repeats the proof of Theorem 2.1] word for word. In this way we obtain the weighted inequality (2.4), restricted to a fixed neighborhood of the lateral surface of the plate $\Omega_{h}$. We next consider the domains $\omega^{k+1}, \ldots, \omega^{L}$ that intersect at least one of the sets $\omega^{1}, \ldots, \omega^{k}$. It can be assumed that $\rho_{h}(y)>c>0$ for $y \in \omega^{k+1} \cup \cdots \cup \omega^{L}$, that is, in (2.3) we can take $\rho_{h}(y) \equiv 1$. Suppose, 
for example, that $\omega^{l} \cap \omega^{m} \neq \varnothing$ and $l \leq k<m \leq L$. We consider the plate $\Omega_{h}^{m}$. Then we have the inequality

$$
\mathbf{|} u ; \Omega_{h}^{m} \mathbf{|}_{1}^{2} \leq c\left(\mathcal{E}\left(u, \Omega_{h}^{m}\right)+\mid u ; \Omega_{h}^{m} \cap \Omega_{h}^{l} \mathbf{|}_{1}^{2}\right),
$$

which together with the inequality $\left|u ; \Omega_{h}^{l}\right|^{2} \leq c \mathcal{E}\left(u, \Omega_{h}^{l}\right)$ gives us the estimate

$$
\left|u ; \Omega_{h}^{m}\right|_{1}^{2} \leq c \mathcal{E}\left(u ; \Omega_{h}^{m} \cup \Omega_{h}^{l}\right) .
$$

Thus, Korn's inequality has been obtained for the elements of the covering $\left\{\omega^{1}, \ldots, \omega^{n}\right\}$ that are neighboring for the elements closest to $\partial \omega$. The next series are handled in the same way as the second. Going through finitely many series establishes the following result.

Theorem 2.2. If $\Omega_{h}$ is a locally periodic plate (1.2) and $u \in \stackrel{\circ}{H^{1}}\left(\Omega_{h}, \Gamma_{h}\right)^{3}$, then Korn's inequality

$$
\left|u ; \Omega_{h}\right|^{2} \leq c \mathcal{E}\left(u ; \Omega_{h}\right)
$$

holds, where the constant $c$ is independent of the field $u$ and the parameter $h \in(0,1]$, and | | $\mid$ is the weighted anisotropic norm (2.3).

\section{Procedure for constructing the formal asymptotics}

3.1. Asymptotic Ansätze and limit problems. We introduce the fast variables

$$
\xi=(\eta, \zeta), \quad \eta=\left(\eta_{1}, \eta_{2}\right), \quad \eta_{i}=y_{i} h^{-1}, \quad \zeta=z h^{-1}
$$

and we regard every function $(x, \xi) \mapsto v(x, \xi)$ as a composite function $x \mapsto v\left(x, h^{-1} x\right)$.

The matrix differential operator $L$ on the left-hand side of the system (1.4) admits the decomposition

$$
\begin{aligned}
L\left(h, x, \nabla_{x}\right)= & D\left(-\nabla_{x}\right)^{\top} A(\xi, x) D\left(\nabla_{x}\right)=-h^{-2} D_{\xi}^{\top} A(\xi, y) D_{\xi} \\
& -h^{-1}\left\{D_{\xi}^{\top} A(\xi, y) D_{y}+D_{y}^{\top} A(\xi, y) D_{\xi}\right\}-h^{0} D_{y}^{\top} A(\xi, y) D_{y} \\
= & : h^{-2} L^{0}\left(y, \xi, \nabla_{\xi}\right)+h^{-1} L^{1}\left(y, \xi, \nabla_{y}, \nabla_{\xi}\right)+h^{0} L^{2}\left(y, \xi, \nabla_{y}\right),
\end{aligned}
$$

where $D_{y}=D\left(\nabla_{y}, 0\right)$ and $D_{\xi}=D\left(\nabla_{\xi}\right)$. The normal $n$ to the surface $\Sigma_{h}$ has the form

$$
n(h, x)=N(h, x)^{-1}\left\{\nu(\xi, y)+h \nu^{0}(\xi, y)\right\},
$$

where $\nu(\cdot, y)$ is the unit outward normal vector to the surface $\Sigma(y)$, the quantity

$$
N=\left(1+2 h \nu^{\top} \nu^{0}+h^{2}\left|\nu^{0}\right|^{2}\right)^{1 / 2}
$$

is a normalizing factor, and $\nu^{0}=\left(\nu_{1}^{0}, \nu_{2}^{0}, 0\right)^{\top}$ is a correction term due to the dependence of the surface on the slow variables $y$ (see Lemma 3.2 below). According to (3.2) the matrix operator $B$ in the boundary conditions (1.5) satisfies the formula

$$
\begin{aligned}
N(h, x) B\left(h, x, \nabla_{x}\right)= & N(h, x) D(n)^{\top} A(\xi, x) D\left(\nabla_{x}\right) \\
= & h^{-1} D(\nu)^{\top} A D_{\xi} \\
& +h^{1}\left\{D(\nu)^{\top} A D_{y}+D\left(\nu^{0}\right)^{\top} A D_{\xi}\right\}+h^{0} D\left(\nu^{0}\right)^{\top} A D_{y} \\
= & : h^{-2} B^{0}\left(y, \xi, \nabla_{\xi}\right)+h^{-1} B^{1}\left(y, \xi, \nabla_{y}, \nabla_{\xi}\right)+h^{0} B^{2}\left(y, \xi, \nabla_{y}\right) .
\end{aligned}
$$

In view of the structure of the matrix $D$ the operators $D_{y}$ and $D_{\zeta}=D\left(0,0, \frac{\partial}{\partial \zeta}\right)$ are connected by an identity that is "crucial" in the theory of thin plates (see [13]):

$$
D_{y} e^{3} w_{3}(y)=\sum_{i=1}^{2} D_{\xi} \zeta e^{i} \partial_{i} w_{3}(y) ;
$$


here $w_{3}$ is an arbitrary function and $e^{i}$ is the unit vector in the direction of the $x_{i}$-axis.

For the right-hand sides of the problem (1.4)-(1.6) we take the standard asymptotic Ansatz (see [12], [13], and others)

$$
\begin{aligned}
& f(h, x)=h^{-1} f^{0}(\xi, y)+h^{0} \bar{f}^{0}(y)+\tilde{f}(h, x), \\
& g(h, y)=h^{0} g^{0}(\xi, y)+\tilde{g}(h, x) .
\end{aligned}
$$

Here the terms $f^{0}, g^{0}$, and $\bar{f}^{0}$ are subject to the relations

$$
\int_{S(y)} f_{3}^{0} d \xi+\int_{\Sigma(y)} g_{3}^{0} d s_{\xi}=0, \bar{f}^{0}(y)=e^{3} \bar{f}_{3}^{0}(y),
$$

and the smallness conditions for the remainders $\tilde{f}$ and $\tilde{g}$ will be given in 4.1 . The vector-valued functions $f^{0}, g^{0}$, and $\bar{f}^{0}$ are assumed to be smooth; the exact smoothness requirements will also be formulated in 4.1 Corresponding to the representations (3.6) is the following asymptotic Ansatz for the solution of the problem (1.4)-(1.6):

$$
h^{-2} U^{-2}(y)+h^{-1} U^{-1}(\xi, y)+h^{0} U^{0}(\xi, y)+h^{1} U^{1}(\xi, y)+\cdots .
$$

The first two terms of the Ansatz (3.8) are known,

$$
U^{-2}(y)=e^{3} w_{3}(y), \quad U^{-1}(y, \xi)=\sum_{i=1}^{2} e^{i}\left(w^{i}(y)-\zeta \frac{\partial w_{3}}{\partial y_{i}}(y)\right),
$$

and the problem for the column $w=\left(w_{1}, w_{2}, w_{3}\right)^{\top}$ is determined in the construction of the subsequent terms. The component $w_{3}$ describes mainly the bending of the plate, while the components $w_{1}$ and $w_{2}$ describe its longitudinal displacements. The obvious fact that a plate is easier to bend than to stretch explains the difference between the powers of $h$ multiplying the functions $w_{3}$ and $w_{1}, w_{2}$ in (3.8).

We substitute the expansions (3.8), (3.6), 3.1), and (3.4) in the equalities (1.4), (1.5) and gather the coefficients of like powers of the parameter $h$. We obtain a set of limit problems, of which the first is

$$
L^{0} U^{-2}=0 \quad \text { in } S(x), \quad B^{0} U^{-2}=0 \quad \text { on } \Sigma(y) .
$$

It is clear that the function $U^{-2}$ in (3.9), which does not depend on the transversal variable $\zeta$, satisfies the equalities 3.10 .

Let us consider the second limit problem

$$
L^{0} U^{-1}=-L^{1} U^{-2} \quad \text { in } S(y), \quad B^{0} U^{-1}=-B^{1} U^{-2} \quad \text { on } \Sigma(y) .
$$

The crucial identity (3.5) and the definitions (3.9) imply the relation

$$
D_{\xi} U^{-1}+D_{y} U^{-2}=0,
$$

which ensures the equalities 3.11).

Using the formulas 3.10-(3.12), we derive the problem for the term $U^{0}$,

$$
\begin{aligned}
L^{0} U^{0} & =D_{\xi}^{\top} A D_{y} U^{-1}+D_{y}^{\top} A\left\{D_{\xi} U^{-1}+D_{y} U^{-2}\right\} \\
& =D_{\xi}^{\top} A D_{y}^{\top} U^{-1} \quad \text { in } S(y), \\
B^{0} U^{0} & =-D(\nu)^{\top} A D_{y} U^{-1}-D\left(\nu^{0}\right)^{\top} A\left\{D_{\xi} U^{-1}+D_{y} U^{-2}\right\} \\
& =-D(\nu)^{\top} A D_{y}^{\top} U^{-1} \quad \text { on } \Sigma(y),
\end{aligned}
$$

which we supplement by the periodicity conditions

$$
\left.U^{0}\right|_{\varphi_{y}\left(\eta_{i}=0\right)}=\left.U^{0}\right|_{\varphi_{y}\left(\eta_{i}=l_{i}\right)},\left.\quad \frac{\partial U^{0}}{\partial \eta_{i}}\right|_{\varphi_{y}\left(\eta_{i}=0\right)}=\left.\frac{\partial U^{0}}{\partial \eta_{i}}\right|_{\varphi_{y}\left(\eta_{i}=l_{i}\right)}, \quad i=1,2
$$


that is, the equalities necessary for the continuity of the function $U^{0}$ and its derivatives in the plate $\Omega_{h}$ must hold on the lateral surface of the cell $S(y)$.

We shall need the Sobolev-Slobodetskiu space $H^{l+\gamma}(\omega)$ with smoothness exponents $l \in \mathbb{N}_{0}$ and $\gamma \in(0,1)$, in which the norm is

$$
\begin{aligned}
\left\|v ; H^{l+\gamma}(\omega)\right\|=\left(\sum_{k=0}^{l} \|\right. & \nabla_{y}^{k} v ; L_{2}(\omega) \|^{2} \\
& \left.\quad+\int_{\omega} \int_{\omega}\left|\left(\nabla_{y}^{l} v\right)(y)-\left(\nabla_{y}^{l} v\right)(\mathbf{y})\right|^{2}|y-\mathbf{y}|^{-2(1+\gamma)} d y d \mathbf{y}\right)^{1 / 2} .
\end{aligned}
$$

Here $\nabla_{y}^{l} v$ is the collection of all partial derivatives of the function $v$ of order $l$. We also introduce the space $H^{l+\gamma}(\omega \longrightarrow \mathfrak{B})$ of abstract functions with values in the Banach space $\mathfrak{B}$; the norm in this space is defined as

$$
\begin{aligned}
\| v ; H^{1+\gamma}(\omega \longrightarrow & \mathfrak{B}) \|=\left(\int_{\omega}\left\{\|v(y, \cdot) ; \mathfrak{B}\|^{2}+\left\|\nabla_{y} v(y, \cdot) ; \mathfrak{B}\right\|^{2}\right\} d y\right. \\
& \left.+\int_{\omega} \int_{\omega}\left\|\left(\nabla_{y} v\right)(y, \cdot)-\left(\nabla_{y} v\right)(\mathbf{y}, \cdot) ; \mathfrak{B}\right\|^{2}|y-\mathbf{y}|^{-2(1+\gamma)} d y d \mathbf{y}\right)^{1 / 2} .
\end{aligned}
$$

As $\mathfrak{B}$ we must take the subspaces $C_{\text {per }}^{s, \alpha}(S(y))$ and $C_{\text {per }}^{s, \alpha}(\Sigma(y))$ of functions in the Hölder classes $C^{s, \alpha}(S(y))$ and $C^{s, \alpha}(\Sigma(y))$ that satisfy the periodicity conditions (3.14). By $H^{l+\gamma}\left(\omega \longrightarrow C_{\mathrm{per}}^{s, \alpha}(S(y))\right)$ we mean the space of functions $v$ for which the composite $v \circ \varphi_{y}$ belongs to $H^{l+\gamma}\left(\omega \longrightarrow C_{\text {per }}^{s, \alpha}(S)\right)$, with

$$
\left\|v ; H^{l+\gamma}\left(\omega \longrightarrow C_{\text {per }}^{s, \alpha}(S(y))\right)\right\|=\left\|v \circ \varphi_{y} ; H^{l+\gamma}\left(\omega \longrightarrow C_{\text {per }}^{s, \alpha}(S)\right)\right\| .
$$

Here $\varphi_{y}$ is the diffeomorphism used in the definitions (1.2).

Finally, the norm in $C_{\text {per }}^{s, \alpha}(S(y))$ is given by the formula

$$
\begin{aligned}
\left\|v ; C^{s, \alpha}(S(y))\right\|=\sum_{k=0}^{s} \sup & \left\{\left|\nabla_{x}^{k} v(x)\right| ; x \in S(y)\right\} \\
& +\sup \left\{|x-\mathbf{x}|^{-\alpha}\left|\left(\nabla_{x}^{s} v\right)(x)-\left(\nabla_{x}^{s} v\right)(\mathbf{x})\right| ; x, \mathbf{x} \in S(y)\right\},
\end{aligned}
$$

and the norm in $C^{s, \alpha}(\Sigma(y))$ is obtained from (3.16) by replacing the domain $S(y)$ with the surface $\Sigma(y)$ and the gradient $\nabla_{x}$ with the tangent gradient $\nabla_{\tau}$.

The following assertion is known (see, for example, [13, Proposition 2.5 .5 (2)]).

Lemma 3.1. The problem

$$
L^{0} U=\Phi \quad \text { in } S(y), \quad B^{0} U=\Psi \quad \text { on } \Sigma(y)
$$

with right-hand side

$$
\{\Phi, \Psi\} \in H^{l+\gamma}\left(\omega \longrightarrow C_{\text {per }}^{s, \alpha}(S(y))^{3} \times C_{\text {per }}^{s, \alpha}(\Sigma(y))^{3}\right), \quad l, s \in \mathbb{N}_{0}, \quad \gamma, \alpha \in(0,1)
$$

has a solution $U \in H^{l+\gamma}\left(\omega \longrightarrow C_{\mathrm{per}}^{s+2, \alpha}(S(y))^{3}\right)$ if and only if the solvability conditions

$$
\int_{S(y)} \Phi_{k}(y, \xi) d \xi+\int_{\Sigma(y)} \Psi_{k}(y, \xi) d s_{\xi}=0, \quad k=1,2,3,
$$

hold for almost all $y \in \omega$. The solution $U$ is determined up to a constant (with respect to $\zeta)$ term, but if it is subject to the orthogonality conditions

$$
\int_{S(y)} U_{k}(y, \xi) d \xi=0, \quad k=1,2,3
$$


then it becomes unique. Such a solution $U$ satisfies the estimate

$$
\begin{aligned}
\| U ; H^{l+\gamma}(\omega \longrightarrow & \left.C_{\text {per }}^{s+2, \alpha}(S(y))^{3}\right) \| \\
& \leq c\left\|\{\Phi, \Psi\} ; H^{l+\gamma}\left(\omega \longrightarrow C_{\text {per }}^{s, \alpha}(S(y))^{3} \times C_{\text {per }}^{s+1, \alpha}(\Sigma(y))^{3}\right)\right\|
\end{aligned}
$$

and inherits from $\Phi$ and $\Psi$ a smooth dependence on the variables $y=\left(y_{1}, y_{2}\right) \in \omega$.

We remark that for any function $\mathcal{W} \in H^{1}(S(y))^{6}$

$$
\begin{aligned}
\int_{S(y)}\left(e^{j}\right)^{\top} D_{\xi}^{\top} \mathcal{W}(\xi) d \xi-\int_{\Sigma(y)}\left(e^{j}\right)^{\top} D(\nu)^{\top} \mathcal{W}(\xi) d s_{\xi} \\
\quad=\int_{S(y)}\left(D_{\xi} e^{j}\right)^{\top} \mathcal{W}(\xi) d \xi=0, \quad j=1,2 .
\end{aligned}
$$

Taking $\mathcal{W}=A D_{y} U^{-1}$, we now see that the right-hand sides in (3.13) satisfy the conditions (3.18), that is, the problem (3.13) is solvable. Moreover, recalling the representations (3.9), we derive the formulas (for details see $\S 6.2$ in [13])

$$
\begin{gathered}
D_{y} U^{-1}(y, \zeta)=\mathcal{Y}(\zeta) \mathcal{D}\left(\nabla_{y}\right) w(y) \\
\mathcal{D}\left(\nabla_{y}\right)=\left(\begin{array}{cccccc}
\partial_{1} & 0 & \alpha \partial_{2} & 0 & 0 & 0 \\
0 & \partial_{2} & \alpha \partial_{1} & 0 & 0 & 0 \\
0 & 0 & 0 & \alpha \partial_{1}^{2} & \alpha \partial_{2}^{2} & \partial_{1} \partial_{2}
\end{array}\right)^{\top}, \\
\mathcal{Y}(\zeta)=\left(\begin{array}{ccc}
\mathbb{I}_{3} & -\alpha \zeta \mathbb{I}_{3} \\
\mathbb{O}_{3} & \mathbb{O}_{3}
\end{array}\right), \quad \alpha=\frac{1}{\sqrt{2}}
\end{gathered}
$$

here $\mathbb{I}_{q}$ and $\mathbb{O}_{q}$ are the $q \times q$ identity and zero matrices.

Thus, the solution $U^{0}$ is representable as follows:

$$
U^{0}(\xi, y)=\mathcal{X}(\xi, y) \mathcal{D}\left(\nabla_{y}\right) w(y)
$$

The $3 \times 6$ matrix $\mathcal{X}$ is periodic and satisfies the relations

$$
\begin{aligned}
-D_{\xi}^{\top} A D_{\xi} \mathcal{X} & =D_{\xi}^{\top} A \mathcal{Y} & & \text { in } S(y), \\
D(\nu)^{\top} A D_{\xi} \mathcal{X} & =-D(\nu)^{\top} A \mathcal{Y} & & \text { on } \Sigma(y) .
\end{aligned}
$$

In other words, the columns $\mathcal{X}^{1}, \ldots, \mathcal{X}^{6}$ of $\mathcal{X}$ are solutions of the problem (3.17) with special right-hand sides. We subject these columns to the orthogonality conditions (3.19); by Lemma 3.1 the matrix $\mathcal{X}$ becomes smoothly dependent on the variable $y \in \bar{\omega}$.

3.2. The resulting problem. We proceed to a consideration of the problem

$$
\begin{array}{ll}
L^{0} U^{1}=-L^{1} U^{0}-L^{2} U^{-1}+f^{0} & \text { in } S(y), \\
B^{0} U^{1}=-B^{1} U^{0}-B^{2} U^{-1}+g^{0} & \text { on } \Sigma(y) .
\end{array}
$$

The following lemma essentially contains a rule for differentiating integrals with variable limits.

Lemma 3.2. For any function $W(\xi, y) \in H^{1}\left(\omega \longrightarrow H_{\mathrm{per}}^{1}(S(y))\right)$

$$
\frac{d}{d y_{i}} \int_{S(y)} W(\xi, y) d \xi=\int_{S(y)} \frac{\partial}{\partial y_{i}} W(\xi, y) d \xi-\int_{\Sigma(y)} \nu_{i}^{0}(\xi, y) W(\xi, y) d s_{\xi}
$$

where $i=1,2$, and $\nu_{i}^{0}$ are the components of the column $\nu^{0}$ in the formula (3.2) for the normal $n$ to the surface $\Sigma_{h}$. 
Proof. It suffices to verify the formula (3.26) separately for the functions $\chi_{j} W$, where $\left\{\chi_{j}\right\}$ is a partition of unity on a neighborhood of the set $\overline{S(y)}$ and is periodic with respect to $\zeta$ and independent of $y$. The supports of the cutoff functions $\chi_{j}$ can be taken so small that for $y \in B_{\rho}$ (a disk of small radius) the intersection $S(y) \cap \operatorname{supp} \chi_{j}$ falls in a set $\Xi^{j}(y)$ which in a suitable system of Cartesian coordinates $\widetilde{\xi}^{j}=\left(\widetilde{\eta}^{j}, \widetilde{\zeta}^{j}\right)$ is given by the formulas

$$
\widetilde{\eta}^{j} \in \widetilde{Q}^{j}, \quad 0<\widetilde{\zeta}^{j}<\widetilde{F}^{j}\left(\widetilde{\eta}^{j}, y\right),
$$

where $\widetilde{Q}^{j}$ is a rectangle, and $\widetilde{F}^{j}$ is a smooth positive function. Furthermore, the intersection $\Sigma(y) \cap \operatorname{supp} \chi_{j}$ is inside the surface

$$
\widetilde{T}^{j}(y)=\left\{\xi: \widetilde{\eta}^{j} \in \widetilde{Q}^{j}, \widetilde{\zeta}^{j}=\widetilde{F}^{j}\left(\widetilde{\eta}^{j}, y\right)\right\} .
$$

We can assume without loss of generality that the system of coordinates $\left(\widetilde{\eta}^{j}, \widetilde{\zeta}^{j}\right)$ coincides with the system $\xi=(\eta, \zeta)$, and we remove the symbols " $"$ and " $j$ " from (3.27) and (3.28).

The normal $n(h, x)$ to the surface $\left\{x: h^{-1} y \in Q, z=h F\left(h^{-1} y, y\right)\right\}$ has the form (3.2), where

$$
\begin{aligned}
\nu(\xi, y) & =N(h, x)^{-1 / 2}\left(\frac{\partial F}{\partial \eta_{1}}\left(h^{-1} y, y\right), \frac{\partial F}{\partial \eta_{2}}\left(h^{-1} y, y\right), 1\right)^{\top}, \\
\nu^{0}(\xi, y) & =N(h, x)^{-1 / 2}\left(\frac{\partial F}{\partial y_{1}}\left(h^{-1} y, y\right), \frac{\partial F}{\partial y_{2}}\left(h^{-1} y, y\right), 0\right)^{\top}, \\
N(h, x) & =1+\left|\frac{\partial F}{\partial \eta_{1}}\left(h^{-1} y, y\right)\right|^{2}+\left|\frac{\partial F}{\partial \eta_{2}}\left(h^{-1} y, y\right)\right|^{2}, \\
N(h, x) & =1+h \sum_{i=1}^{2} \frac{\partial F}{\partial y_{i}}\left(h^{-1} y, y\right)\left(\frac{\partial F}{\partial \eta_{i}}\left(h^{-1} y, y\right)+h \frac{\partial F}{\partial y_{i}}\left(h^{-1} y, y\right)\right) .
\end{aligned}
$$

Keeping the notation $W$ for the product $\chi_{i} W$, we have

$$
\begin{aligned}
\frac{d}{d y_{i}} \int_{\Xi(y)} W(\xi, y) d \xi & =\int_{Q} \frac{d}{d y_{i}} \int_{0}^{F(\eta, y)} W(\eta, \zeta, y) d \zeta d \eta \\
& =\int_{\Xi(y)} \frac{\partial}{\partial y_{i}} W(\xi, y) d \xi+\int_{Q} \frac{\partial F}{\partial y_{i}}(\eta, y) W(\eta, F(\eta, y), y) d \eta
\end{aligned}
$$

It remains to observe that according to $(3.29)_{2,3}$ the last (double) integral is equal to

$$
\begin{aligned}
\int_{Q} \frac{\partial F}{\partial y_{i}} & (\eta, y)\left(1+\left|\nabla_{\eta} F(\eta, y)\right|^{2}\right)^{-1 / 2} W(\eta, F(\eta, y), y)\left(1+\left|\nabla_{\eta} F(\eta, y)\right|^{2}\right)^{1 / 2} d \eta \\
& =\int_{T(y)} \frac{\partial F}{\partial y_{i}}(\eta, y)\left(1+\left|\nabla_{\eta} F(\eta, y)\right|^{2}\right)^{-1 / 2} W(\xi, y) d s_{\eta}=-\int_{T(y)} \nu_{i}^{0} W(\xi, y) d s_{\eta} .
\end{aligned}
$$

By the identity (3.26) and the assumption (3.6), the right-hand sides of the problem (3.25),

$$
\begin{aligned}
& F^{1}=D_{\xi}^{\top} A D_{y} U^{0}+D_{y}^{\top} A\left\{D_{\xi} U^{0}+D_{y} U^{-1}\right\}+f^{0}, \\
& G^{1}=-D(\nu)^{\top} A D_{y} U^{0}-D\left(\nu^{0}\right)^{\top} A\left\{D_{\xi} U^{0}+D_{y} U^{-1}\right\}+g^{0},
\end{aligned}
$$


satisfy the condition (3.18) for $k=3$. Indeed, integrating by parts and using the formulas (3.21) and (3.5), (3.7), we find that

$$
\begin{aligned}
\int_{S(y)} F_{3}^{1}(y, \xi) d \xi+\int_{\Sigma(y)} G_{3}^{1}(y, \xi) d s_{\xi} \\
=\left(e^{3}\right)^{\top}\left(\int_{S(y)} D_{\xi}^{\top} A D_{y} U^{0} d \xi-\int_{\Sigma(y)} D(\nu)^{\top} A D_{y} U^{0} d s_{\xi}\right) \\
+\left(e^{3}\right)^{\top}\left(\int_{S(y)} D_{y}^{\top} A\left\{D_{\xi} U^{0}+D_{y} U^{-1}\right\} d \xi-\int_{\Sigma(y)} D\left(\nu^{0}\right)^{\top} A\left\{D_{\xi} U^{0}+D_{y} U^{-1}\right\} d s_{\xi}\right) \\
=\left(e^{3}\right)^{\top} D_{y}^{\top} \int_{S(y)} A\left\{D_{\xi} U^{0}+D_{y} U^{-1}\right\} d \xi \\
=\sum_{i=1}^{2}\left(e^{i}\right)^{\top} \frac{\partial}{\partial y_{i}} \int_{S(y)} D_{\xi}^{\top} A\left\{D_{\xi} U^{0}+D_{y} U^{-1}\right\} d \xi \\
=\sum_{i=1}^{2} \frac{\partial}{\partial y_{i}} \int_{S(y)}\left(D_{\xi} \zeta e^{i}\right)^{\top} A\left\{D_{\xi} U^{0}+D_{y} U^{-1}\right\} d \xi \\
=-\sum_{i=1}^{2} \frac{\partial}{\partial y_{i}}\left(e^{i}\right)^{\top}\left(\int_{S(y)} \zeta D_{\xi}^{\top} A\left\{D_{\xi} U^{0}+D_{y} U^{-1}\right\} d \xi\right. \\
\left.\quad+\int_{\Sigma(y)} \zeta D(\nu)^{\top} A\left\{D_{\xi} U^{0}+D_{y} U^{-1}\right\} d s_{\xi}\right)=0 .
\end{aligned}
$$

The last equality holds in view of (3.11).

The other two solvability conditions in (3.18), $k=i=1,2$, for the right-hand sides in (3.30) can be written in the form

$$
\begin{aligned}
0 & =\left(e^{i}\right)^{\top}\left(\int_{S(y)} D_{y}^{\top} A \mathcal{Z} d \xi+\int_{\Sigma(y)} D\left(\nu^{0}\right)^{\top} A \mathcal{Z} d s_{\xi}\right) \mathcal{D}\left(\nabla_{y}\right) w+\mathcal{F}_{i} \\
& =\left(e^{i}\right)^{\top} D_{y}^{\top} \int_{S(y)} A \mathcal{Z} d \xi \mathcal{D}\left(\nabla_{y}\right) w+\mathcal{F}_{i}, \quad i=1,2,
\end{aligned}
$$

where, by (3.22) and (3.23),

$$
\begin{gathered}
D_{\zeta} U^{0}+D_{y} U^{-1}=\mathcal{Z} \mathcal{D}\left(\nabla_{y}\right) w, \quad \mathcal{Z}(y)=D_{\xi} \mathcal{X}(y, \xi)+\mathcal{Y}(\zeta) \\
\mathcal{F}_{i}(y)=\int_{S(y)} f_{i}^{0}(y, \xi) d \xi+\int_{\Sigma(y)} g_{i}^{0}(y, \xi) d s_{\xi}, \quad i=1,2 .
\end{gathered}
$$

Thus, the pair of differential equations (3.33) has been obtained for the column $w$. To get another equation, we consider the residual left in the system (1.4 -1.6) by the sum $\mathbf{U}$ of four terms in (3.8). Using (3.1) and (3.6), we have

$$
\begin{gathered}
L \mathbf{U}-f=h^{-4} L^{0} U^{-2}+h^{-3}\left(L^{0} U^{-1}+L^{1} U^{-2}\right)+h^{-2}\left(L^{0} U^{0}+L^{1} U^{-1}+L^{2} U^{-2}\right) \\
+h^{-1}\left(L^{0} U^{1}+L^{1} U^{0}+L^{2} U^{-1}-f^{0}\right)+h^{0}\left(L^{1} U^{1}+L^{2} U^{0}-\bar{f}^{0}\right)+h^{1} L^{2} U^{1}-\tilde{f} .
\end{gathered}
$$

The vectors $U^{-2}, \ldots, U^{1}$ were chosen so that the coefficients of $h^{-4}, \ldots, h^{-1}$ vanish. Consequently, repeating the transformations in connection with the boundary conditions 
(1.5), we arrive at the formulas

$$
\begin{aligned}
& L \mathbf{U}-f=-h^{0} f^{1}-\left\{\tilde{f}-h^{1} L^{2} U^{1}\right\}, \\
& B \mathbf{U}-g=-h^{1} g^{1}-\left\{\widetilde{g}-\left[N^{-1}-1\right]\left(g^{0}+h^{1} g^{1}\right)-h^{2} B^{2} U^{1}\right\},
\end{aligned}
$$

in which

$$
\begin{aligned}
f^{1} & =\mathbf{f}+D_{\xi}^{\top} A D_{y} U^{1}, & g^{1} & =\mathbf{g}-D^{\top}\left(\widetilde{\nu}^{0}\right) A D_{y} U^{1}, \\
\mathbf{f} & =D_{y}^{\top} A\left(D_{\xi} U^{1}+D_{y} U^{0}\right)+\bar{f}^{0}, & \mathbf{g} & =-D^{\top}(\nu) A\left(D_{\xi} U^{1}+D_{y} U^{0}\right) .
\end{aligned}
$$

Since $1-N(h, y)^{-1}=O\left(h^{2}\right)$ by virtue of (3.3), the expressions in the curly brackets in (3.34) can be put in the remainder. The terms $h^{0} f^{1}$ and $h^{1} g^{1}$ are analogous to the components $h^{-1} f^{0}$ and $h^{0} g^{0}$ in the formulas (3.6); ; therefore, it is natural to subject them to an orthogonality condition like (3.7):

$$
\int_{S(y)} f_{3}^{1}(y, \xi) d \xi+\int_{\Sigma(y)} g_{3}^{1}(y, \xi) d s_{\xi}=0, \quad y \in w .
$$

Using (3.21), (3.26), and (3.35), we transform the left-hand side of (3.36) to the form

$$
\int_{S(y)} \mathbf{f}_{3} d \xi+\int_{\Sigma(y)} \mathbf{g}_{3} d s_{\xi}=\bar{f}_{3}^{0} \operatorname{meas}_{3} S(y)+\left(e^{3}\right)^{\top} D_{y}^{\top} \int_{S(y)} A\left(D_{\xi} U^{1}+D_{y} U^{0}\right) d \xi
$$

We denote the last term by $I(y)$. In view of the key identity (3.5), we get, using integration by parts, the relation

$$
\begin{aligned}
& I(y)= \sum_{i=1}^{2} \frac{\partial}{\partial y_{i}} \int_{S(y)}\left(D_{\xi} \zeta e^{i}\right)^{\top} A\left(D_{\xi} U^{1}+D_{y} U^{0}\right) d \xi \\
&=\sum_{i=1}^{2} \frac{\partial}{\partial y_{i}}\left(e^{i}\right)^{\top}\left[-\int_{S(y)} \zeta D_{\xi}^{\top} A\left(D_{\xi} U^{1}+D_{y} U^{0}\right) d \xi\right. \\
& \\
&\left.\quad+\int_{\Sigma(y)} \zeta D\left(\nu^{0}\right)^{\top} A\left(D_{\xi} U^{1}+D_{y} U^{0}\right) d s_{\xi}\right] .
\end{aligned}
$$

Since in view of the equalities (3.25)

$$
\begin{aligned}
D_{\xi}^{\top} A D_{\xi} U^{1}+D_{\xi}^{\top} A D_{y} U^{0} & =-L^{0} U^{1}-L^{1} U^{0}-D_{y}^{\top} A D_{\xi} U^{0} \\
& =L^{2} U^{-1}-f^{0}-D_{y}^{\top} A D_{\xi} U^{0} \\
& =-D_{y}^{\top} A D_{y} U^{-1}-D_{y}^{\top} A D_{\xi} U^{0}-f^{0} \\
& =-D_{y}^{\top} A\left(D_{y} U^{-1}+D_{\xi} U^{0}\right)-f^{0}, \\
D\left(\nu^{0}\right)^{\top} A D_{\xi} U^{1}+D\left(\nu^{0}\right)^{\top} A D_{y} U^{0} & =B^{0} U^{1}+B^{1} U^{0}-D\left(\nu^{0}\right)^{\top} A D_{\xi} U^{0} \\
& =-B^{2} U^{-1}+g^{0}-D\left(\nu^{0}\right)^{\top} A D_{\xi} U^{0} \\
& =-D\left(\nu^{0}\right)^{\top} A D_{y} U^{-1}-D\left(\nu^{0}\right)^{\top} A D_{\xi} U^{0}+g^{0} \\
& =-D\left(\nu^{0}\right)^{\top} A\left(D_{y} U^{-1}+D_{\xi} U^{0}\right)+g^{0},
\end{aligned}
$$

we transform $I(y)$ as follows:

$$
\begin{aligned}
& I(y)=\sum_{i=1}^{2} \frac{\partial}{\partial y_{i}}\left[\left(e^{i}\right)^{\top} \int_{S(y)} \zeta D_{y}^{\top} A\left(D_{\xi} U^{0}+D_{y} U^{-1}\right) d \xi\right. \\
& \left.-\int_{\Sigma(y)} \zeta D\left(\nu^{0}\right)^{\top} A\left(D_{\xi} U^{0}+D_{y} U^{-1}\right) d s_{\xi}+\int_{S(y)} \zeta f^{0}(y, \xi) d \xi+\int_{\Sigma(y)} \zeta g^{0}(y, \xi) d s_{\xi}\right] .
\end{aligned}
$$


We now apply Lemma 3.2 to the function

$$
W(\xi, y)=\zeta A\left(D_{\xi} U^{0}+D_{y} U^{-1}\right)=\zeta A\left(D_{\xi} \mathcal{X}+\mathcal{Y}\right) \mathcal{D}\left(\nabla_{y}\right) w(y),
$$

and obtain the equality

$$
\begin{aligned}
& \left(e^{i}\right)^{\top} D_{y}^{\top} \int_{S(y)} \zeta A\left(D_{\xi} \mathcal{X}+\mathcal{Y}\right) d \xi \mathcal{D}\left(\nabla_{y}\right) w \\
& =\left(e^{i}\right)^{\top} \int_{S(y)} \zeta D_{y}^{\top} A\left(D_{\xi} U^{0}+D_{y} U^{-1}\right) d \xi-\int_{\Sigma(y)} \zeta D\left(\nu^{0}\right)^{\top} A\left(D_{\xi} U^{0}+D_{y} U^{-1}\right) d s_{\xi} .
\end{aligned}
$$

This gives us the formula

$$
I(y)=\sum_{i=1}^{2}\left(e^{i}\right)^{\top} D_{y}^{\top} \frac{\partial}{\partial y_{i}} \int_{S(y)} \zeta A\left(D_{\xi} \mathcal{X}+\mathcal{Y}\right) d \xi \mathcal{D}\left(\nabla_{y}\right) w+\sum_{i=1}^{2} \frac{\partial}{\partial y_{i}} \mathcal{F}_{i}^{\prime},
$$

where

$$
\begin{aligned}
& \mathcal{F}_{i}^{\prime}(y)=\int_{S(y)} \zeta f_{i}^{0}(y, \xi) d \xi+\int_{\Sigma(y)} \zeta g_{i}^{0}(y, \xi) d s_{\xi}, \\
& \mathcal{F}_{3}(y)=\bar{f}_{3}^{0}(y) \operatorname{meas}_{3} S(y)+\sum_{i=1}^{2} \frac{\partial}{\partial y_{i}} \mathcal{F}_{i}^{\prime}(y) .
\end{aligned}
$$

Accordingly, the condition (3.36) is equivalent to the differential equation

$$
\sum_{i=1}^{2}\left(e^{i}\right)^{\top} D_{y}^{\top} \frac{\partial}{\partial y_{i}} \int_{S(x)} \zeta A \mathcal{Z} d \xi \mathcal{D}\left(\nabla_{y}\right) w+\mathcal{F}_{3}=0 .
$$

Thus, the system of equations (3.32, (3.39) is obtained for the unknown column $w$; it is called the resulting system.

We note that the rows $D_{y} e^{1}, D_{y} e^{2}, D_{y}\left(e^{1} \partial_{1}+e^{2} \partial_{2}\right) \zeta$ in (3.32) and (3.39) are rows of the matrix $\mathcal{D}\left(\nabla_{y}\right)^{\top} \mathcal{Y}(\zeta)^{\top}$. Therefore, the resulting problem can be written in the short divergence form

$$
\mathcal{D}\left(-\nabla_{y}\right)^{\top} \mathcal{M}(y) \mathcal{D}\left(\nabla_{y}\right) w(y)=\mathcal{F}(y), \quad y \in w .
$$

Here $\mathcal{F}=\left(\mathcal{F}_{1}, \mathcal{F}_{2}, \mathcal{F}_{3}\right)^{\top}$ is the column with components (3.33), (3.38), and the $6 \times 6$ matrix-valued function $\mathcal{M}$ is defined by

$$
\begin{aligned}
\mathcal{M}(y) & =\int_{S(y)} \mathcal{Y}(\zeta)^{\top} A(y, \xi)\left(D_{\xi} \mathcal{X}(y, \xi)+\mathcal{Y}(\zeta)\right) d \xi \\
& =\int_{S(y)}\left(D_{\xi} \mathcal{X}(y, \xi)+\mathcal{Y}(\zeta)\right) A(y, \xi)\left(D_{\xi} \mathcal{X}(y, \xi)+\mathcal{Y}(\zeta)\right) d \xi
\end{aligned}
$$

The last transformation is obtained by integration by parts with the use of the relations (3.24) for $\mathcal{X}$.

Since the lateral surface of the plate is rigidly clamped (see (1.6)), we supplement the system (3.40) by the Dirichlet boundary conditions

$$
w(y)=0, \quad \partial_{\mathbf{n}} w_{3}(y)=0, \quad y \in \partial \omega,
$$

where $\mathbf{n}=\left(\mathbf{n}_{1}, \mathbf{n}_{2}\right)^{\top}$ is the outward unit normal vector to the contour $\partial \omega$ and $\partial_{\mathbf{n}}=\mathbf{n}^{\top} \nabla_{y}$ is differentiation along $\mathbf{n}$. The proof of the following assertion can be found, for example, in [13, Lemma 6.4.1 and Theorem 4.1.4]. 
Theorem 3.1. For all $y \in \omega$ the matrix $\mathcal{M}(y)$ is symmetric and positive definite.

2) If $\mathcal{F} \in H^{l-1}(\omega)^{2} \times H^{l-2}(\omega)$ for some $l \in\{1,2, \ldots\}$, then there exists a unique solution $w \in H^{l+1}(\omega)^{2} \times H^{l+2}(\omega)$ of the problem (3.40), (3.42) and it satisfies the estimate

$$
\left\|w ; H^{l+1}(\omega)^{2} \times H^{l+2}(\omega)\right\| \leq c_{l}\left\|\mathcal{F} ; H^{l-1}(\omega)^{2} \times H^{l-2}(\omega)\right\| .
$$

\section{Justification OF THE ASYMPtotics}

4.1. Asymptotic approximation. We assume that for some $\gamma \in(0,1 / 2]$ the terms in the decompositions (3.6) satisfy the inclusions

$$
\begin{aligned}
& f^{0} \in H^{1+\gamma}\left(\omega \longrightarrow C_{\mathrm{per}}^{0, \alpha}(S(y))^{3}\right), \\
& g^{0} \in H^{1+\gamma}\left(\omega \longrightarrow C_{\mathrm{per}}^{1, \alpha}(\Sigma(y))^{3}\right), \\
& \bar{f}_{3}^{0} \in H^{\gamma}(\omega),
\end{aligned}
$$

and the remainders satisfy the inclusions

$$
\tilde{f} \in L_{2}\left(\Omega_{h}\right)^{3}, \quad \widetilde{g} \in L_{2}\left(\Sigma_{h}\right)^{3} .
$$

Let

$$
\begin{gathered}
\widetilde{\mathcal{N}}_{\gamma-1 / 2}=h^{1 / 2-\gamma}\left[\sum_{i=1}^{2}\left(\left\|\rho_{h} \widetilde{f}_{i} ; L_{2}\left(\Omega_{h}\right)\right\|+h^{-1 / 2}\left\|\rho_{h} \widetilde{g}_{i} ; L_{2}\left(\Sigma_{h}\right)\right\|\right)\right. \\
\left.+h^{-1}\left\|\rho_{h}^{2} \widetilde{f}_{3} ; L_{2}\left(\Omega_{h}\right)\right\|+h^{-3 / 2}\left\|\rho_{h}^{2} \widetilde{g}_{3} ; L_{2}\left(\Sigma_{h}\right)\right\|\right], \\
\mathcal{N}_{1+\gamma}=\left\|\bar{f}_{3}^{0} ; H^{\gamma}(\omega)\right\|+\left\|\left\{f^{0}, g^{0}\right\} ; H^{1+\gamma}\left(\omega \longrightarrow C_{\text {per }}^{0, \alpha}(S(y))^{3} \times C_{\text {per }}^{1, \alpha}(\Sigma(y))^{3}\right)\right\| ;
\end{gathered}
$$

here $H^{1+\gamma}(\omega \longrightarrow \ldots)$ is the Sobolev-Slobodetskiu space of abstract functions with the norm

$$
\begin{gathered}
\left\|\left\{f^{0}, g^{0}\right\} ; H^{1+\gamma}(\omega \longrightarrow \ldots)\right\| \\
=\left[\int_{\omega}\left(\left\|\left\{f^{0}(y, \cdot), g^{0}(y, \cdot)\right\} ; \ldots\right\|^{2}+\left\|\left\{\nabla_{y} f^{0}(y, \cdot), \nabla_{y} g^{0}(y, \cdot)\right\} ; \ldots\right\|^{2}\right) d y\right. \\
\quad+\int_{\omega} \int_{\omega} \|\left\{\left(\nabla_{y} f^{0}\right)(y, \cdot)-\left(\nabla_{y} f^{0}\right)(\mathbf{y}, \cdot),\left(\nabla_{y} g^{0}\right)(y, \cdot)\right. \\
\left.\left.-\left(\nabla_{y} g^{0}\right)(\mathbf{y}, \cdot)\right\} ; \ldots \|^{2}|y-\mathbf{y}|^{-2(1+\gamma)} d y d \mathbf{y}\right]^{1 / 2}
\end{gathered}
$$

(the triple dots replace the range). We assume that the quantity $\widetilde{\mathcal{N}}_{\gamma-1 / 2}$ has unit order $h^{0}$ in comparison with the small parameter $h$, and the quantity $\mathcal{N}_{1+\gamma}$ does not depend on the parameter in general.

The formulas $(3.33)_{2}$ and $(3.38)_{2}$ show that in view of $(4.1)_{1,2}$ the right-hand side $\mathcal{F}$ of the system (3.40) falls in the space $H^{1+\gamma}(\omega)^{2} \times H^{\gamma}(\omega)$, and

$$
\left\|\mathcal{F} ; H^{1+\gamma}(\omega)^{2} \times H^{\gamma}(\omega)\right\| \leq c \mathcal{N}_{1+\gamma} .
$$


Indeed, for the function $\mathcal{F}_{3}$ in $(3.38)_{2}$ the fragment of norm $\left\|\mathcal{F}_{3} ; H^{\gamma}(\omega)\right\|$ (see (3.15) with $l=0)$ can be estimated as follows:

$$
\begin{aligned}
& \int_{\omega} \int_{\omega}\left|\mathcal{F}_{3}(y)-\mathcal{F}_{3}(\mathbf{y})\right|^{2}|y-\mathbf{y}|^{-2(1+\gamma)} d y d \mathbf{y} \\
& \leq c \int_{\omega} \int_{\omega}\left\{\sum _ { i = 1 } ^ { 2 } \left[\int_{S}\left|\left(\nabla_{y} f_{i}^{0}\right)(y, \xi)-\left(\nabla_{y} f_{i}^{0}\right)(\mathbf{y}, \xi)\right|^{2} d \xi\right.\right. \\
& \left.\left.\quad+\int_{\Sigma}\left|\left(\nabla_{y} g_{i}^{0}\right)(y, \xi)-\left(\nabla_{y} g_{i}^{0}\right)(\mathbf{y}, \xi)\right|^{2} d s_{\xi}\right]+\left|f_{3}^{0}(y)-f_{3}^{0}(\mathbf{y})\right|^{2}\right\}|y-\mathbf{y}|^{-2(1+\gamma)} d y d \mathbf{y} \\
& \leq c\left(\sum _ { i = 1 } ^ { 2 } \int _ { \omega } \int _ { \omega } \left[\sup \left\{\left|\left(\nabla_{y} f_{i}^{0}\right)(y, \xi)-\left(\nabla_{y} f_{i}^{0}\right)(\mathbf{y}, \xi)\right|: \xi \in S\right\}^{2}\right.\right. \\
& \left.\quad+\sup \left\{\left|\left(\nabla_{y} g_{i}^{0}\right)(y, \xi)-\left(\nabla_{y} g_{i}^{0}\right)(\mathbf{y}, \xi)\right|: \xi \in \Sigma\right\}^{2}\right]|y-\mathbf{y}|^{-2(1+\gamma)} d y d \mathbf{y} \\
& \left.+\left\|\bar{f}_{3}^{0} ; H^{\gamma}(\omega)\right\|^{2}\right) .
\end{aligned}
$$

The last integral does not exceed the square of the norm (4.3) because of the presence of the term $\sup |v|$ in the norm (3.16). The analogous inequality holds for the remaining part of the expression $\left\|\mathcal{F}_{3} ; H^{\gamma}(\omega)\right\|^{2}$,

$$
\begin{aligned}
&\left\|\mathcal{F}_{3} ; L_{2}(\omega)\right\|=\int_{\omega}\left|\mathcal{F}_{3}(y)\right|^{2} d y \\
& \leq c \int_{\omega}\left\{\sum_{i=1}^{2}\left[\int_{S}\left|\left(\nabla_{y} f_{i}^{0}\right)(y, \xi)\right|^{2} d \xi+\int_{\Sigma}\left|\left(\nabla_{y} g_{i}^{0}\right)(y, \xi)\right|^{2} d s_{\xi}\right]+\left|f_{3}^{0}(y)\right|^{2}\right\} d y \\
& \leq c\left(\sum _ { i = 1 } ^ { 2 } \int _ { \omega } \left[\sup \left\{\left|\left(\nabla_{y} f_{i}^{0}\right)(y, \xi)\right|: \xi \in S\right\}^{2}\right.\right. \\
&\left.\left.\quad+\sup \left\{\left|\left(\nabla_{y} g_{i}^{0}\right)(y, \xi)\right|: \xi \in \Sigma\right\}^{2}\right]+\left\|\bar{f}_{3}^{0} ; H^{\gamma}(\omega)\right\|^{2}\right) .
\end{aligned}
$$

The norms $\left\|\mathcal{F}_{i} ; H^{1+\gamma}(\omega)\right\|^{2}$ for the functions $\mathcal{F}_{i}$ in $\left({ }^{3.33}\right)_{2}$ can be estimated in exactly the same way.

The solution $w \in H^{2}(\omega)^{2} \times H^{3}(\omega)$ of the problem (3.40), (3.42) satisfies the inequality

$$
\left\|w ; H^{3+\gamma}(\omega)^{2} \times H^{4+\gamma}(\omega)\right\| \leq c\left\|\mathcal{F} ; H^{1+\gamma}(\omega)^{2} \times H^{\gamma}(\omega)\right\|
$$

(see 35, 36 and other references). By the formulas (3.9), (3.23), (4.4), (4.5) and Lemma 3.1, applied to the problem 3.25, we conclude that

$$
\begin{array}{r}
U^{i} \in H^{\gamma+2-j}\left(\omega \longrightarrow C_{\mathrm{per}}^{2, \alpha}(S)^{3}\right), \quad j=-2,-1,0,1, \\
\left\|U^{j} ; H^{\gamma+2-j}\left(\omega \longrightarrow C_{\mathrm{per}}^{2, \alpha}(S)^{3}\right)\right\| \leq c \mathcal{N}_{1+\gamma} .
\end{array}
$$

We define two asymptotic approximations to the solution $u(h, x)$ of the problem (1.4)(1.6):

$$
\begin{aligned}
\mathfrak{U}^{0}(h, x) & =h^{-2} U^{-2}(y)+h^{-1} U^{-1}(\zeta, y)+h^{0} U^{0}(\xi, y), \\
\mathfrak{U}_{X}^{0}(h, x) & =h^{-2} U^{-2}(y)+h^{-1} U^{-1}(\zeta, y)+h^{0} X_{h}(y) U^{0}(\xi, y),
\end{aligned}
$$

where $X_{h}(y)$ is a cutoff function equal to zero in the $c h$-neighborhood $\mathcal{V}_{c h}$ of the contour $\partial \omega$ and equal to 1 for $y \in \omega \backslash \mathcal{V}_{2 c h}$. It is clearly possible to ensure the inequality

$$
\left|\nabla_{y}^{k} X_{h}(y)\right| \leq c_{k} h^{-k}, \quad k \in \mathbb{N}_{0} .
$$


In view of (3.42) the first two terms of the Ansatz (3.8) satisfy the boundary conditions

$$
U^{-2}(y)=0, \quad U^{-1}(\zeta, y)=0, \quad x \in \Gamma_{h} ;
$$

therefore, only the third term in $(4.7))_{2}$ is multiplied by the cutoff function. The functions

$$
x \mapsto U^{-2}(y), \quad U^{-1}\left(h^{-1} z, y\right), \quad U^{0}\left(h^{-1} x, y\right)
$$

belong to the space $H^{1}\left(\Omega_{h}\right)^{3}$. For the functions $U^{-2}$ and $U^{-1}$ what is claimed is obvious, since they are polynomials in the variable $z$ with coefficients in $H^{4+\gamma}(\omega)^{3}$ and $H^{3+\gamma}(\omega)^{3}$, respectively. Since $\mathcal{D}\left(\nabla_{y}\right) w \in H^{2+\gamma}(\omega)^{6}$ and the solution $\mathcal{X}$ of the problem (3.24) is a smooth periodic (with respect to $\eta$ ) matrix on the set $\{(y, \xi): y \in \bar{\omega}, \xi \in \overline{S(y)}\}$, it follows that $U^{0}=\mathcal{X} \mathcal{D}\left(\nabla_{y}\right) w \in H^{2+\gamma}\left(\Omega_{h}\right)^{3}$. By construction, the approximation $\mathfrak{U}_{X}^{0}$ satisfies the boundary condition (1.6), that is, $\mathfrak{U}_{X}^{0} \in \stackrel{\circ}{H}^{1}\left(\Omega_{h}, \Gamma_{h}\right)^{3}$. The following assertion is essentially known (see [13, Lemma 6.2.1]).

Lemma 4.1. If the right-hand sides $f^{0}, g^{0}$ are subject to the inclusions (4.1) and the orthogonality condition (3.7), and the expressions (3.9) and (3.23) are constructed from the solution $w$ of the resulting problem (3.40), (3.42), then the functions (4.7) are connected by the inequality

$$
\left|\mathfrak{U}^{0}-\mathfrak{U}_{X}^{0} ; \Omega_{h}\right|+\left\|D\left(\nabla_{x}\right) \mathfrak{U}_{X}^{0}-D\left(\nabla_{x}\right) \mathfrak{U}^{0} ; L_{2}\left(\Omega_{h}\right)\right\| \leq c h^{0} \mathcal{N}_{1+\gamma},
$$

in which the constant $c$ is independent of $f^{0}, g^{0}$ and $h \in(0,1]$.

4.2. Computation and estimation of residuals. We substitute the approximation $\mathfrak{U}_{X}^{0}$ in the relations $(1.4)-(1.6)$ and transform the residuals obtained, taking account of the equalities (3.10), (3.11) and (3.13), (3.25):

$$
\begin{aligned}
L \mathfrak{U}_{X}^{0}-f= & L\left(X_{h}-1\right) U^{0}+L \mathfrak{U}^{0}-f \\
= & L\left(X_{h}-1\right) U^{0}+h^{-4} L^{0} U^{-2} \\
& +h^{-3}\left(L^{0} U^{-1}+L^{1} U^{-2}\right)+h^{-2}\left(L^{0} U^{0}+L^{1} U^{-1}+L^{2} U^{-2}\right) \\
& +h^{-1}\left(L^{1} U^{0}+L^{2} U^{-1}-f^{0}\right)+h^{0}\left(L^{2} U^{0}-\bar{f}^{0}\right)-\widetilde{f} \\
= & L\left(X_{h}-1\right) U^{0}-h^{-1} L^{0} U^{1}+h^{0}\left(L^{2} U^{0}-\bar{f}^{0}\right)-\widetilde{f} \\
B \mathfrak{U}_{X}^{0}-g= & B\left(X_{h}-1\right) U^{0}+B \mathfrak{U}^{0}-g \\
= & B\left(X_{h}-1\right) U^{0}+h^{-3} N^{-1} B^{0} U^{-2} \\
& +h^{-2} N^{-1}\left(B^{0} U^{-1}+B^{1} U^{-2}\right)+h^{-1}\left(B^{0} U^{0}+B U^{-1}\right) \\
& +h^{0} N^{-1}\left(B^{1} U^{0}-g^{0}\right)+\left(N^{-1}-1\right) g^{0}-\widetilde{g} \\
= & B\left(X_{h}-1\right) U^{0}-h^{0} N^{-1} B^{0} U^{1}+\left(N^{-1}-1\right) g^{0}-\widetilde{g} .
\end{aligned}
$$

Consequently, the difference $\mathcal{R}=u-\mathfrak{U}_{X}^{0}$ satisfies the problem

$$
\begin{aligned}
& L \mathcal{R}=L\left(1-X_{h}\right) U^{0}+h^{-1} L^{0} U^{1}-h^{0}\left(L^{2} U^{0}-\bar{f}^{0}\right)+\tilde{f} \quad \text { in } \Omega_{h}, \\
& B \mathcal{R}=B\left(1-X_{h}\right) U^{0}+h^{0} N^{-1} B^{0} U^{1}+\left(1-N^{-1}\right) g^{0}+\widetilde{g} \text { on } \Sigma_{h}, \\
& \mathcal{R}=0 \quad \text { on } \Gamma_{h} \text {. }
\end{aligned}
$$

Let us take the inner product of the system (4.10) 1 with $\mathcal{R}$ and integrate by parts in $\Omega_{h}$, using the boundary conditions $4.10{ }_{2,3}$. As a result we get the equality

$$
\left(A D\left(\nabla_{x}\right) \mathcal{R}, D\left(\nabla_{y}\right) \mathcal{R}\right)_{\Omega_{h}}=\widetilde{I}+I_{X}+I_{1}-I_{0}
$$


where

$$
\begin{aligned}
\widetilde{I} & =(\widetilde{f}, \mathcal{R})_{\Omega_{h}}+(\widetilde{g}, \mathcal{R})_{\Sigma_{h}}+\left(\left[1-N^{-1}\right] g^{0}, \mathcal{R}\right)_{\Sigma_{h}}, \\
I_{X} & =\left(L\left(1-X_{h}\right) U^{0}, \mathcal{R}\right)_{\Omega_{h}}+\left(B\left(1-X_{h}\right) U^{0}, \mathcal{R}\right)_{\Sigma_{h}} \\
& =\left(A D\left(\nabla_{x}\right)\left(1-X_{h}\right) U^{0}, D\left(\nabla_{x}\right) \mathcal{R}\right)_{\Omega_{h}} \\
I^{0} & =\left(L^{2} U^{0}-\bar{f}^{0}, \mathcal{R}\right)_{\Omega_{h}} \\
I^{1} & =h^{-1}\left(L^{0} U^{1}, \mathcal{R}\right)_{\Omega_{h}}+\left(B^{0} U^{1}, \mathcal{R}\right)_{\Sigma_{h}}
\end{aligned}
$$

Since the formula (3.3) ensures the relation

$$
\left|1-N^{-1}(h, y)\right| \leq c h^{2},
$$

we estimate the term 4.12$)_{1}$, recalling the definition $[2.3$ of the norm $|\bullet|$ and the notation (4.3):

$$
\begin{aligned}
|\widetilde{I}| \leq c h^{\gamma-1 / 2} \widetilde{N}_{\gamma-1 / 2}\left\{h\left\|\rho_{h}^{-2} \mathcal{R}_{3} ; L_{2}\left(\Omega_{h}\right)\right\|+h^{3 / 2}\left\|\rho_{h}^{-2} \mathcal{R}_{3} ; L_{2}\left(\Sigma_{h}\right)\right\|\right. \\
\left.+\sum_{i=1}^{2}\left(\left\|\rho_{h}^{-1} \mathcal{R}_{i} ; L_{2}\left(\Omega_{h}\right)\right\|+h^{1 / 2}\left\|\rho_{h}^{-1} \mathcal{R}_{i} ; L_{2}\left(\Sigma_{h}\right)\right\|\right)\right\} \\
\leq c h^{\gamma-1 / 2} \widetilde{N}_{\gamma-1 / 2}\left|\mathcal{R} ; \Omega_{h}\right|^{2} .
\end{aligned}
$$

Let $\omega(h)=\omega \cap \mathcal{V}_{2 l h}$ and $\Omega(h)=\left\{x \in \Omega_{h}: y \in \omega(h)\right\}$. If the function $w$ satisfies the relations (3.42), then we have the estimates

$$
\begin{array}{rlrl}
h^{p-2}\left\|\nabla_{y}^{p} w^{3} ; L^{2}(\omega(h))\right\| & \leq c h^{1 / 2}\left\|w_{3} ; H^{3}(\omega)\right\|, & & p=0,1,2, \\
h^{q-1}\left\|\nabla_{y}^{q} w_{i} ; L_{2}(\omega(h))\right\| \leq c h^{1 / 2}\left\|w_{i} ; H^{2}(\omega)\right\|, & q=0,1, \quad i=1,2 .
\end{array}
$$

The following formula expresses the rule for differentiating the composite function $x \longmapsto U\left(y, h^{-1} x\right)$ :

$$
D\left(\nabla_{x}\right) U\left(y, h^{-1} x\right)=\left.\left\{h^{-1} D_{\xi} U(y, \xi)+D_{y} U(y, \xi)\right\}\right|_{\xi=h^{-1} x} .
$$

Using the estimate (4.8) and the formula (4.16) for the smooth matrix $\mathcal{X}$ in the representation (3.24), we get by the inequalities (4.15) that

$$
\begin{aligned}
\left|I_{X}\right| & \leq c\left\|\varepsilon(\mathcal{R}) ; L_{2}\left(\Omega_{h}\right)\right\|\left\{h^{-1}\left\|\mathcal{D}\left(\nabla_{y}\right) w ; L_{2}(\Omega(h))\right\|+\left\|\nabla_{y} \mathcal{D}\left(\nabla_{y}\right) w ; L_{2}\left(\Omega_{h}(h)\right)\right\|\right\} \\
& \leq c \mathcal{E}\left(\mathcal{R} ; \Omega_{h}\right)^{1 / 2} h^{1 / 2}\left\{h^{-1}\left\|\mathcal{D}\left(\nabla_{y}\right) w ; L_{2}(\omega(h))\right\|+\left\|\nabla_{y} \mathcal{D}\left(\nabla_{y}\right) w ; L-2(\omega(h))\right\|\right\} \\
& \leq c \mathcal{E}\left(\mathcal{R} ; \Omega_{h}\right)^{1 / 2} h^{1 / 2}\left\{h^{-1} h^{1 / 2}+1\right\}\left\|w ; H^{2}(\omega)^{2} \times H^{3}(\omega)\right\| \\
& \leq c h^{0} \mathcal{N}_{1+\gamma} \mathcal{E}\left(\mathcal{R} ; \Omega_{h}\right)^{1 / 2} .
\end{aligned}
$$

In the treatment of $I_{1}$ it is necessary to make the transformations

$$
\begin{aligned}
I_{1} & =-\left(h^{-1} D_{\xi}^{\top} A D_{\xi} U^{1}, \mathcal{R}\right)_{\Omega_{h}}+\left(D(\nu)^{\top} A D_{\xi} U^{1}, \mathcal{R}\right)_{\Sigma_{h}} \\
& =-\left(D\left(\nabla_{x}\right)^{\top} A D_{\xi} U^{1}, \mathcal{R}\right)_{\Omega_{h}}+\left(D(\nu)^{\top} A D_{\xi} U^{1}, \mathcal{R}\right)_{\Sigma_{h}}-I_{10}, \\
I_{10} & =-\left(D_{y}^{\top} A D_{\xi} U^{1}, \mathcal{R}\right)_{\Omega_{h}} .
\end{aligned}
$$

To estimate $I_{1}$ we need the following result. 
Lemma 4.2. Suppose that $y_{0} \in \omega$ and $V \in L_{2}\left(\omega \longrightarrow L_{\infty}\left(S\left(y_{0}\right)\right)\right)$. Then the function $x \longrightarrow v(x)=V\left(y, h^{-1} x\right)$ belongs to $L_{2}\left(\Omega_{h}\right)$, and

$$
\left\|v ; L_{2}\left(\Omega_{h}\right)\right\| \leq \operatorname{ch}^{1 / 2}\left\|V ; L_{2}\left(\omega \longrightarrow L_{\infty}\left(S\left(y_{0}\right)\right)\right)\right\| .
$$

Proof. Let $S_{h}\left(y_{0}\right)=\left\{x: h^{-1} x \in S\left(y_{0}\right)\right\}$ be the $h$-contraction of the cell $S\left(y_{0}\right)$, and let $Q_{h}\left(y_{0}\right)=\left\{y \in \omega: h^{-1} x \in S\left(y_{0}\right)\right\}$ be the projection of $S_{h}\left(y_{0}\right)$ on the plane $z=0$. We extend $V$ by zero for $y \in \mathbb{R}^{2} \backslash \omega$ and verify the inequality (4.19):

$$
\begin{aligned}
\left\|v ; L_{2}\left(\Omega_{h}\right)\right\|^{2} & =\int_{\Omega_{h}}\left|V\left(y, \frac{y}{h}, \frac{z}{h}\right)\right|^{2} d y d z=\int_{\omega}\left(\int_{S_{h}\left(y_{0}\right)}\left|V\left(y, \frac{y}{h}, \frac{z}{h}\right)\right|^{2} d y d z\right) d y_{0} \\
& \leq \int_{\omega}\left(\int_{S_{h}\left(y_{0}\right)}\left|\sup \left\{|V(y, \xi)|: \xi \in S\left(y_{0}\right)\right\}\right|^{2} d y d z\right) d y_{0} \\
& \leq c \int_{\omega} h\left(\int_{Q_{h}\left(y_{0}\right)}\left\|V(y, .) ; L_{\infty}\left(S\left(y_{0}\right)\right)\right\|^{2} d y\right) d y_{0} \\
& =\operatorname{ch}\left\|V ; L_{2}\left(\omega \longrightarrow L_{\infty}\left(S\left(y_{0}\right)\right)\right)\right\|^{2} .
\end{aligned}
$$

By (4.6),

$$
\nabla_{\xi} U^{0} \in H^{2+\gamma}\left(\omega \longrightarrow C_{\text {per }}^{1, \alpha}(S(y))^{3 \times 3}\right) \subset L_{2}\left(\omega \longrightarrow L_{\infty}(S(y))^{3 \times 3}\right)
$$

for $j=1$, and therefore it follows from the estimate (4.19) that

$$
\begin{aligned}
\left|I_{1}+I_{10}\right| & \leq c\left\|D_{\xi} U^{0} ; L_{2}\left(\Omega_{h}\right)\right\| \times\left\|D\left(\nabla_{x}\right) \mathcal{R} ; L_{2}\left(\Omega_{h}\right)\right\| \\
& \leq \operatorname{ch}^{1 / 2}\left\|U^{0} ; H^{2+\gamma}\left(\omega \longrightarrow C_{\mathrm{per}}^{1, \alpha}(S(y))\right)\right\| \times\left\|e(\mathcal{R}) ; L_{2}\left(\Omega_{h}\right)\right\| \\
& \leq \operatorname{ch}^{1 / 2} \mathcal{N}_{1+\gamma} \mathcal{E}\left(\mathcal{R} ; \Omega_{h}\right)^{1 / 2} .
\end{aligned}
$$

We combine the inner product in (4.18) 2 with $I_{0}$ and get that

$$
\begin{gathered}
I_{0}+I_{10}=\sum_{j=1}^{3} \int_{\Omega_{h}} \mathcal{F}_{j}\left(y, \frac{x}{h}\right) \mathcal{R}_{j}(x) d x=: \sum_{j=1}^{3} J_{3}, \\
\mathcal{F}(y, \xi)=D_{y}^{\top} A(y, \xi)\left\{D_{\xi} U^{1}(y, \xi)+D_{y} U^{0}(y, \xi)\right\}-\bar{f}^{0}(y) .
\end{gathered}
$$

According to (4.1) 2 and (4.6) we have $\mathcal{F} \in H^{\gamma}\left(\omega \longrightarrow C_{\text {per }}^{1, \alpha}\left(S\left(y_{0}\right)\right)^{3}\right)$, and

$$
\left\|\mathcal{F} ; H^{\gamma}\left(\omega \longrightarrow C_{\text {per }}^{1, \alpha}\left(S\left(y_{0}\right)\right)\right)\right\| \leq c \mathcal{N}_{1+\gamma} .
$$

Using the formulas (3.21), (3.10) and (3.11), (3.13), (3.25), we rewrite the equality (3.37) in the form

$$
\int_{S\left(y_{0}\right)} \mathcal{F}(y, \xi) d \xi=0, \quad y \in \omega .
$$

Let us estimate the integrals $J_{i}$ in $4.211_{1}$. In view of the definition of the norm $|\bullet|$ we have

$$
\left\|\mathcal{R}_{i} ; L_{2}\left(\Omega_{h}\right)\right\| \leq c\left|\mathcal{R} ; \Omega_{h}\right|, \quad i=1,2,
$$

and thus it follows from Lemma 4.2 that

$$
\left|J_{i}\right| \leq \operatorname{ch}^{1 / 2}\left\|\mathcal{F}_{i} ; L_{2}\left(\omega \longrightarrow C_{\text {per }}^{0, \alpha}\left(S\left(y_{0}\right)\right)\right)\right\| \times\left\|\mathcal{R}_{i} ; L_{2}\left(\Omega_{h}\right)\right\| \leq c h^{1 / 2} \mathcal{N}_{1+\gamma}\left|\mathcal{R} ; \Omega_{h}\right| .
$$

The analogous inequality for $\mathcal{R}_{3}$ contains the factor $h^{-1}$ :

$$
\left\|\mathcal{R}_{3} ; L_{2}\left(\Omega_{h}\right)\right\|+\left\|\nabla_{x} \mathcal{R}_{3} ; L_{3}\left(\Omega_{h}\right)\right\| \leq c h^{-1}\left|\mathcal{R} ; \Omega_{h}\right| .
$$


Therefore, it is somewhat more involved to estimate the integral $J_{3}$. We choose a point $y_{0} \in \omega$ and a cell $S_{h}(y)$ intersecting $\Omega_{h}$. Extending $\mathcal{R}$ by zero to $S_{h}\left(y_{0}\right)$, we get that

$$
\begin{gathered}
J_{3}=\int_{S_{h}\left(y_{0}\right)} \mathcal{F}_{3}\left(y, \frac{x}{h}\right)\left(\mathcal{R}_{3}(x)-\overline{\mathcal{R}}_{3}\right) d x+\overline{\mathcal{R}}_{3} \int_{S_{h}\left(y_{0}\right)} \mathcal{F}_{3}\left(y, \frac{x}{h}\right) d x \\
\overline{\mathcal{R}}_{3}=\left[\operatorname{meas}_{3} S_{h}(y)\right]^{-1} \int_{S_{h}\left(y_{0}\right)} \mathcal{R}_{3} d x
\end{gathered}
$$

The following formulas are consequences of Poincaré's inequality:

$$
\begin{aligned}
\left\|\mathcal{R}_{3}-\overline{\mathcal{R}}_{3} ; L_{2}\left(S_{h}\left(y_{0}\right)\right)\right\| & \leq \operatorname{ch}\left\|\nabla_{x}\left(\mathcal{R}_{3}-\overline{\mathcal{R}_{3}}\right) ; L_{2}\left(S_{h}\left(y_{0}\right)\right)\right\| \\
& =\operatorname{ch}\left\|\nabla_{x} \mathcal{R}_{3} ; L_{2}\left(S_{h}\left(y_{0}\right)\right)\right\|, \\
\left|\overline{\mathcal{R}_{3}}\right| & \leq \operatorname{ch}^{-3} \int_{S_{h}\left(y_{0}\right)}\left|\mathcal{R}_{3}(x)\right| d x \leq c h^{-3 / 2}\left\|\mathcal{R}_{3} ; L_{2}\left(S_{h}\left(y_{0}\right)\right)\right\| .
\end{aligned}
$$

Using Cauchy's inequality $2 a b \leq \varepsilon^{-1} a^{2}+\varepsilon b^{2}$, we now deduce from (4.25) and (4.27) 1 the estimates

$$
\begin{aligned}
\left|\int_{S_{h}\left(y_{0}\right)} \mathcal{F}\left(\mathcal{R}-\overline{\mathcal{R}_{3}}\right) d x\right| \leq c\left(\varepsilon^{-1} \int_{S_{h}\left(y_{0}\right)} \mathcal{F}_{3}^{2} d x+\varepsilon \int_{S_{h}\left(y_{0}\right)}\left(\mathcal{R}_{3}-\overline{\mathcal{R}_{3}}\right)^{2} d x\right) \\
\leq c\left(h \varepsilon^{-1}\left\|\mathcal{F}_{3} ; L_{2}\left(Q_{h}\left(y_{0}\right) \longrightarrow C_{\mathrm{per}}^{0, \alpha}\left(S\left(y_{0}\right)\right)\right)\right\|^{2}+\varepsilon\left|\mathcal{R}^{0} ; S_{h}\left(y_{0}\right)\right|^{2}\right) .
\end{aligned}
$$

We consider the last integral in 4.26 $)_{1}$ and integrate in addition with respect to $\mathbf{y} \in Q_{h}\left(y_{0}\right)$, obtaining the integral

$$
\left[\operatorname{meas}_{2} Q_{h}\left(y_{0}\right)\right]^{-1} \int_{Q_{h}\left(y_{0}\right)} \int_{S_{h}\left(y_{0}\right)} \mathcal{F}_{3}\left(\mathbf{y}, h^{-1} x\right) d x d \mathbf{y}
$$

which is equal to zero by virtue of (4.23). Using the inequality $(4.27)_{2}$, we now find that

$$
\begin{aligned}
\left|\overline{\mathcal{R}_{3}} \int_{S_{h}\left(y_{0}\right)} \mathcal{F}_{3}\left(y, \frac{x}{h}\right) d x\right| \\
\leq\left[\operatorname{meas}_{2} Q_{h}\right]^{-1}\left|\overline{\mathcal{R}_{3}}\right| \int_{Q_{h}\left(y_{0}\right)} \int_{S_{h}\left(y_{0}\right)}\left|\mathcal{F}_{3}\left(y, \frac{x}{h}\right)-\mathcal{F}_{3}\left(\mathbf{y}, \frac{x}{h}\right)\right| d x d y \\
\leq c h^{-2} h^{-3 / 2}\left\|\mathcal{R}_{3} ; L_{2}\left(S_{h}\left(y_{0}\right)\right)\right\| h^{5 / 2} \\
\quad \times\left(\int_{Q_{h}\left(y_{0}\right)} \int_{S_{h}\left(y_{0}\right)}\left|\mathcal{F}_{3}\left(y, \frac{x}{h}\right)-\mathcal{F}_{3}\left(\mathbf{y}, \frac{x}{h}\right)\right|^{2} d x d y\right)^{1 / 2} .
\end{aligned}
$$

We estimate the integrand by the quantity

$$
\sup \left\{\left|\mathcal{F}_{3}(y, \xi)-\mathcal{F}_{3}(\mathbf{y}, \xi)\right|^{2}: \xi \in S\left(y_{0}\right)\right\}
$$

Since $|y-\mathbf{y}| \leq h c\left(l_{1}, l_{2}\right)$ for $y, \mathbf{y} \in Q_{h}\left(y_{0}\right)$, if we multiply the integrand by the factor $|y-\mathbf{y}|^{-2-2 \gamma}$, then we can multiply the majorant by the factor $\left(h^{2+2 \gamma}\right)^{1 / 2}$. One more pair of factors $h$ and $h^{-1}$ appears as a result of integrating with respect to $z$ and applying 
the formula (4.25). Thus,

$$
\begin{aligned}
& \left|\overline{\mathcal{R}_{3}} \int_{S_{h}\left(y_{0}\right)} \mathcal{F}_{3}\left(y, \frac{x}{h}\right) d x\right| \leq c h^{-1} h^{-1}\left|\mathcal{R} ; S_{h}\left(y_{0}\right)\right| h^{1+\gamma} \\
& \quad \times\left(h \int_{Q_{h}\left(y_{0}\right)} \int_{S_{h}\left(y_{0}\right)} \sup \left\{\left|\mathcal{F}_{3}(y, \xi)-\mathcal{F}_{3}(\mathbf{y}, \xi)\right|^{2}: \xi \in S\left(y_{0}\right)\right\}|y-\mathbf{y}|^{-2-2 \gamma} d y d \mathbf{y}\right)^{1 / 2} \\
& \quad \leq c\left(\varepsilon\left|\mathcal{R} ; S_{h}\left(y_{0}\right)\right|^{2}+\varepsilon^{-1} h^{2 \gamma-1}\left\|\mathcal{F}_{3} ; H^{\gamma}\left(Q_{h}\left(y_{0}\right) \longrightarrow C_{\text {per }}^{0, \alpha}\left(S\left(y_{0}\right)\right)\right)\right\|^{2}\right) .
\end{aligned}
$$

We sum the inequalities (4.28) and (4.30) with respect to $y_{0} \in \omega$, that is, with respect to all cells $S_{h}\left(y_{0}\right)$ intersecting $\Omega_{h}$. The summation of the expressions $\varepsilon\left|\mathcal{R} ; S_{h}\left(y_{0}\right)\right|^{2}$ gives $\varepsilon\left|\mathcal{R} ; \Omega_{h}\right|^{2}$, and the result of summation of the expressions containing $\mathcal{F}_{3}$ does not exceed the quantity

$$
\begin{array}{rl}
\varepsilon^{-1} & h \int_{\omega}\left\|\mathcal{F}_{3}(y, .) ; C_{\mathrm{per}}^{0, \alpha}\left(S\left(y_{0}\right)\right)\right\|^{2} d y \\
& +\varepsilon^{-1} h^{2 \gamma-1} \int_{\omega} \int_{\omega}\left\|\mathcal{F}_{3}(y, .)-\mathcal{F}_{3}(\mathbf{y}, .) ; C_{\mathrm{per}}^{0, \alpha}\left(S\left(y_{0}\right)\right)\right\|^{2}|y-\mathbf{y}|^{-2-2 \gamma} d y d \mathbf{y} \\
& \leq \varepsilon^{-1} h^{2 \gamma-1}\left\|\mathcal{F}_{3} ; H^{\gamma}\left(\omega \longrightarrow C_{\text {per }}^{0, \alpha}\left(S\left(y_{0}\right)\right)\right)\right\|^{2} \leq c \varepsilon^{-1} h^{2 \gamma-1} \mathcal{N}_{1+\gamma}^{2} .
\end{array}
$$

Thus,

$$
\left|I_{1}+I_{10}\right| \leq c\left(\varepsilon\left|\mathcal{R} ; \Omega_{h}\right|^{2}+\varepsilon^{-1} h^{2 \gamma-1} \mathcal{N}_{1+\gamma}^{2}\right) .
$$

\subsection{Theorem on the asymptotics.}

Theorem 4.1. Suppose that the right-hand sides $f$ and $g$ satisfy the relations (3.6), (3.7) and (4.1), (4.3). Then the solution $u$ of the problem (1.4)-(1.6) and the asymptotic approximation (4.7) 1 of it are connected by the inequality

$$
\left|u-\mathfrak{U}^{0} ; \Omega_{h}\right|+\left\|D\left(\nabla_{x}\right)(u)-D\left(\nabla_{x}\right)\left(\mathfrak{U}^{0}\right) ; L_{2}\left(\Omega_{h}\right)\right\| \leq c h^{\gamma-1 / 2}\left(\mathcal{N}_{1+\gamma}+\mathcal{N}_{\gamma-1 / 2}\right),
$$

in which the constant $c$ is independent of $h \in(0,1]$ and of the components $f^{0}, g^{0}, \bar{f}^{0}$ and $\tilde{f}, \widetilde{g}$ of the right-hand sides of the problem.

Proof. In 4.2 we established the estimates (4.14), (4.15), (4.20), (4.31) for the terms in (4.11), and in view of them and Korn's inequality (which is ensured by Theorem 2.2) we have the relation

$$
\begin{aligned}
\left|\mathcal{R} ; \Omega_{h}\right|^{2}+\mathcal{E}\left(\mathcal{R} ; \Omega_{h}\right) \leq c\left[h^{\gamma-1 / 2}\left(\widetilde{\mathcal{N}}_{\gamma-1 / 2}+\mathcal{N}_{1+\gamma}\right)\right. & \left(\left|\mathcal{R} ; \Omega_{h}\right|+\mathcal{E}\left(\mathcal{R} ; \Omega_{h}\right)^{1 / 2}\right) \\
& \left.+\varepsilon\left|\mathcal{R} ; \Omega_{h}\right|^{2}+\varepsilon^{-1} h^{2 \gamma-1} \mathcal{N}_{1+\gamma}^{2}\right] .
\end{aligned}
$$

We choose $\varepsilon$ to be small, and after simple transformations we obtain the inequality (4.32), with $\mathfrak{U}_{X}^{0}$ instead of $\mathfrak{U}^{0}$. Using the estimate (4.9), we replace $\mathfrak{U}_{X}^{0}$ by $\mathfrak{U}^{0}$ and arrive at (4.32).

We consider separately the fields of strains, stresses, and displacements in a locally periodic plate $\Omega_{h}$.

Since it is obvious that

$$
D\left(\nabla_{x}\right) U\left(y, h^{-1} x\right)=\left.\left\{h^{-1} D_{\xi} U(y, \xi)+D_{y} U(y, \xi)\right\}\right|_{\xi=h^{-1} x}
$$


for the functions $x \longmapsto U\left(y, h^{-1} x\right)$, the formulas (3.9), (3.22) 1 , (3.23), (3.12) give us that

$$
\begin{aligned}
\varepsilon\left(\mathfrak{U}^{0}\right)= & D\left(\nabla_{x}\right) \mathfrak{U}^{0}=h^{-3} D_{\xi} U^{-2}+h^{-2}\left(D_{\xi} U^{-1}+D_{y} U^{-2}\right) \\
& +h^{-1}\left(D_{\xi} U^{0}+D_{y} U^{-1}\right)+h^{0} U^{0} \\
= & h^{-1}\left(D_{\xi} \mathcal{X}+\mathcal{Y}\right) \mathcal{D}\left(\nabla_{y}\right) w+D_{y} U^{0} .
\end{aligned}
$$

By Lemma 4.2 and the formula (4.6),

$$
\left\|D_{y} U^{0} ; L_{2}\left(\Omega_{h}\right)\right\| \leq c h^{1 / 2}\left\|U^{0} ; L^{2}\left(\omega \longrightarrow C_{\mathrm{per}}^{0, \alpha}(S(y))\right)\right\| \leq c h^{1 / 2} \mathcal{N}_{1+\gamma},
$$

and this together with (4.32) yields the following result.

Corollary 4.1. Under the conditions of Theorem 4.1

$$
\left\|\varepsilon(u)-h^{-1}\left(D_{\xi} \mathcal{X}+\mathcal{Y}\right) \mathcal{D}\left(\nabla_{y}\right) w ; L_{2} \Omega_{h}\right\| \leq c h^{\gamma-1 / 2}\left(\mathcal{N}_{1+\gamma}+\mathcal{N}_{\gamma-1 / 2}\right) .
$$

Multiplying by the stiffness matrix $A(y, \xi)$, we transform 4.35 into an inequality for the stresses:

$$
\left\|\sigma(u)-A\left(D_{\xi} \mathcal{X}+\mathcal{Y}\right) \mathcal{D}\left(\nabla_{y}\right) w ; L_{2} \Omega_{h}\right\| \leq c h^{\gamma-1 / 2}\left(\mathcal{N}_{1+\gamma}+\mathcal{N}_{\gamma-1 / 2}\right) .
$$

We now deal with the displacements.

Corollary 4.2. Under the conditions of Theorem 4.1

$$
\begin{aligned}
h \| \rho_{h}^{-2}\left\{u_{3}-\right. & \left.h^{-2} w_{3}\right\} ; L_{2}\left(\Omega_{h}\right) \| \\
+\sum_{i=1}^{2} \| \rho_{h}^{-1}\left\{u_{i}-h^{-1}\left(w_{i}-\frac{z}{h} \frac{\partial w_{3}}{\partial y_{i}}\right)\right\} & ; L_{2}\left(\Omega_{h}\right) \| \\
& \leq \operatorname{ch}^{\gamma-1 / 2}\left(\mathcal{N}_{1+\gamma}+\mathcal{N}_{\gamma-1 / 2}\right) .
\end{aligned}
$$

Proof. To derive the estimate (4.36) it suffices to use the weighted norm $U^{0}$. With this goal we use integration along the contour $\partial \omega$ to transform the variant of Hardy's inequality

$$
\int_{0}^{d}(\rho+h)^{-2} V^{2}(\rho) d \rho \leq c \int_{0}^{d}\left(\left|\frac{d V}{d \rho}(\rho)\right|^{2}+V^{2}(\rho)\right) d \rho \quad \forall V \in H^{1}(0, d)
$$

into the inequality

$$
h \int_{\omega} \rho_{h}^{-2}|v|^{2} d y \leq c_{\omega} \int_{\omega}\left(\left|\nabla_{y} v\right|^{2}+|v|^{2}\right) d y \quad \forall v \in H^{1}(\omega)
$$

(see Proposition 1.2.3 in [13]). From this, using the relations (4.5) and (4.4), we get the chain of relations

$$
\begin{aligned}
h^{2}\left\|\rho_{h}^{-2} U_{3}^{0} ; L_{2}\left(\Omega_{h}\right)\right\|^{2}+\sum_{i=1}^{2}\left\|\rho_{h}^{-1} U_{i}^{0} ; L_{2}\left(\Omega_{h}\right)\right\|^{2} \leq \operatorname{ch} \int_{\omega}\left(h^{2} \rho_{h}^{-4}+\rho_{h}^{-2}\right)\left|\mathcal{D}\left(\nabla_{y}\right) w\right|^{2} d y \\
\leq \operatorname{ch} \int_{\omega} \rho_{h}^{-2}\left(\left|\nabla_{y} w^{\prime}\right|^{2}+\left|\nabla_{y}^{2} w_{3}\right|^{2}\right) d y \leq\left\|w ; H^{2}(\omega)^{2} \times H^{3}(\omega)\right\|^{2} \leq c \mathcal{N}_{1+\gamma}^{2}
\end{aligned}
$$

which concludes the proof of the corollary. 


\section{REFERENCES}

[1] D. Morgenstern, Herleitung der Plattentheorie aus der dreidemensionalen Elastizitätstheorie, Arch. Rational Mech. Anal. 4 (1959), 145-152. MF 0111252 (22:2116)

[2] B. A. Shoikhet, On the asymptotics of the exact equations of thin plates with composite structure, Prikl. Mat. Mekh. 37 (1973), 914-924; English transl. in Appl. Math. Mech. 37 (1973), 867-877. MR.0349110 (50:1604)

[3] B. A. Shoikhet, An energy identity in physically nonlinear elasticity, and an error estimate for the equations of plates, Prikl. Mat. Mekh. 40 (1976), 317-326; English transl. in Appl. Math. Mech. 40 (1976), 291-301.

[4] P. G. Ciarlet and P. Destuynder, A justification of the two-dimensional linear plate model, J. Mécanique 18 (1979), 315-344. MR0533827 (80e:73046)

[5] P. Destuynder, Comparaison entre les modèles tridimensionnels et bidimensionnels de plaques en élasticité, RAIRO Modél. Math. Anal. Numér. 15 (1981), 331-369. MR0642497 (83h:73069)

[6] S. N. Leora, S. A. Nazarov, and A. V. Proskura, Derivation of the limit equations for elliptic problems in thin domains with the aid of a computer, Zh. Vychisl. Mat. i Mat. Fiz. 26 (1986), 1032-1048; English transl. in U.S.S.R. Comput. Math. and Math. Phys. 26 (1986), no. 7, 47-58. MR0851753 (87j:65155)

[7] B. Miara, Optimal spectral approximation in linearized plate theory, Appl. Anal. 31 (1989), 291-307. MR.1017518 (90g:73078)

[8] P. G. Ciarlet, Plates and junctions in elastic multi-structures: an asymptotic analysis, R. M. A., Vol. 14, Masson and Springer-Verlag, Paris and Heidelberg, 1990. MR1071376 (91h:73034)

[9] A. Mielke, On the justification of plate theories in linear elasticity theory using exponential decay estimates, J. Elasticity 38 (1995), 165-208. MR1336037 (96g:73016)

[10] P. G. Ciarlet, Mathematical elasticity, Vol. II. Theory of plates, Vol. 27, Studies in Mathematics and its Applications, Elsevier, Amsterdam-New York, 1997. MR.1477663 (99e:73001)

[11] O. V. Motygin and S. A. Nazarov, Justification of the Kirchhoff hypotheses and error estimation for two-dimensional models of asymptotic and inhomogeneous plates, including laminated plates, IMA J. Appl. Math. 65 (2000), 1-28. MR.1773872 (2001e:74058)

[12] S. A. Nazarov, Asymptotic analysis of an arbitrarily anisotropic plate of variable thickness (sloping shell), Mat. Sb. 191 (2000), no. 7, 129-159; English transl. in Sbornik: Math. 191 (2000), 10751106. MR.1809932(2001h:74053)

[13] S. A. Nazarov, Asymptotic theory of thin plates and rods, Vol. 1, Reduction of dimension and integral estimates, "Nauchnaya Kniga", Novosibirsk, 2002. (Russian)

[14] D. Caillerie, Plaques élastiques mines à structure périodique de période et d'épaisseur comparables, C. R. Acad. Sc. Paris Sér. II 294 (1982), 159-162. MR0654232 (83d:73046)

[15] D. Caillerie, Thin elastic and periodic plates, Math. Methods Appl. Sci. 6 (1984), 159-191. MR.0751739 (86c:73020)

[16] R. Kohn and M. Vogelius, A new model for thin plates with rapidly varying thickness, Internat. J. Solids Structures 20 (1984), 333-350.

[17] R. Kohn and M. Vogelius, A new model for thin plates with rapidly varying thickness. II. A convergence proof, Quart. Appl. Math. 43 (1985), no. 1, 1-22. MR.782253 (86h:73028)

[18] R. Kohn and M. Vogelius, A new model for thin plates with rapidly varying thickness. III. Comparison of different scalings, Quart. Appl. Math. 44 (1986), no. 1, 35-48. MF0840441 (87j:73065)

[19] N.S. Bakhvalov and G. P. Panasenko, Averaging of processes in periodic media, "Nauka", Moscow, 1984; English transl., Homogenization: Averaging of processes in periodic media, Kluwer, Dordrecht, 1989. MR0797571 (86m:73049)

[20] G. P. Panasenko and M.V. Reztsov, Averaging of the three-dimensional problem of the theory of elasticity in a nonhomogeneous plate, Dokl. Akad. Nauk SSSR 294 (1987), 1061-1065; English transl. in Soviet Math. Dokl. 35 (1987), 630-634. MR0898314 (88h:73015)

[21] T. Lewiński, Effective models of composite periodic plates. I. Asymptotic solution, Internat. J. Solids Structures 27 (1991), 1155-1172. MR1087930 (92c:73081a)

[22] S. A. Nazarov, A general procedure for averaging self-adjoint elliptic systems in multidimensional domains, including thin domains, Algebra i Analiz 7 (1995), no. 5, 1-92; English transl. in St. Petersburg Math. J. 7 (1996), 681-748. MR1365812 (97c:35013)

[23] I. Aganović, E. Marušić-Paloka, and Z. Tutek, Slightly wrinkled plate, Asymptotic Anal. 13 (1996), 1-29. MR 1406165 (97j:73041)

[24] I. Aganović, M. Jurak, E. Marušić-Paloka, and Z. Tutek, Moderately wrinkled plate, Asymptotic Anal. 16 (1998), 273-297. MR.1612817|(99d:73053)

[25] T. Lewiński and J. J. Telega, Plates, laminates, and shells. Asymptotic analysis and homogenization, World Scientific, Singapore-London, 2000. MR1758600(2002e:74001) 
[26] G. A. Chechkin and D. Ciorănescu, Vibration of a thin plate with "rough" surface, Nonlinear Partial Differential Equations and their Applications (Collège de France Seminar, Vol. XIV), Vol. 31, Studies in Mathematics and its Applications, Elsevier, Amsterdam-London-New York, 2002, pp. 147-169. MR.1933963 (2003e:35003)

[27] S. A. Nazarov, Korn's inequalities that are asymptotically sharp for thin domains, Vestnik S.Peterburg. Univ. 1992, no. 8, 19-24; English transl. in Vestnik St. Petersburg Univ. Math. 25 (1992), no. 2, 18-22. MR 1280920 (95m:35026)

[28] G. A. Chechkin and E. A. Pichugina, Weighted Korn's inequality for a thin plate with a rough surface, Russian J. Math. Phys. 7 (2000), no. 3, 279-287. MR.1832718 (2002c:74043)

[29] E. A. Akimova, S. A. Nazarov, and G. A. Chechkin, Weighted Korn's inequality: the "tetris" procedure for an arbitrary periodic plate, Dokl. Akad. Nauk 380 (2001), 439-442; English transl. in Doklady Math. 64 (2001), 205-207. MR1875497

[30] G. A. Iosif'yan [Yosifian], O. A. Oleŭnik, and A. S. Shamaev, Mathematical problems of the theory of strongly inhomogeneous elastic media, Izdat. Moskov. Gos. Univ., Moscow, 1990; English transl., Mathematical problems in elasticity and homogenization, North-Holland, Amsterdam, 1992. MR.1115306 (92i:73009)

[31] K. O. Friedrichs, On the boundary-value problems of the theory of elasticity and Korn's inequality, Ann. Math. 48 (1947), 447-471. MR0022750 (9:255b)

[32] J. Nečas, Les méthodes directes en théorie des équations elliptiques, Masson, Paris, 1967. MR.0227584 (37:3168)

[33] G. Duvaut and J.-L. Lions, Les inéquations en méchanique et en physique, Dunod, Paris, 1972; English transl., Springer-Verlag, Berlin-New York, 1976; Russian transl., "Nauka", Moscow, 1980. MR0464857 (57:4778)

[34] V.A. Kondrat'ev and O. A. Oleĭnik, Boundary-value problems for the system of elasticity theory in unbounded domains. Korn's inequalities, Uspekhi Mat. Nauk 43 (1988), no. 5, 55-98; English transl. in Russian Math. Surveys 43 (1988), no. 5, 65-119. MR0971465 (89m:35061)

[35] C.B. Morrey, Multiple integrals in the calculus of variations, Springer-Verlag, Berlin-New York, 1966. MR0202511 (34:2380)

[36] Ya. A. Roitberg, Elliptic boundary-value problems in spaces of distributions, Kluwer, Dordrecht, 1996. MR 1423135(97m:35055)

Moscow State University, Department of Mechanics and Mathematics, Moscow 119899 , Russia

E-mail address: chechkin@mech.math.msu.su

St. Petersburg State University, St. Petersburg, Russia

E-mail address: serna@snark.ipme.ru

Moscow State University, Department of Mechanics and Mathematics, Moscow 119899 , RUSSIA

E-mail address: chechkin@mech.math.msu.su

Translated by H. H. MCFADEN 